\title{
General framework for multiple scattering of polarized waves including anisotropies and Berry phase
}

\author{
V. Rossetto ${ }^{*}$ \\ Université Joseph Fourier, Grenoble, France \\ and LPMMC-CNRS Maison des Magistères, BP 166, 25 Avenue des Martyrs, 38042 Grenoble CEDEX 09, France
}

(Received 3 June 2009; revised manuscript received 10 August 2009; published 24 November 2009)

\begin{abstract}
We develop a framework for the multiple scattering of a polarized wave. We consider particles with spin propagating in a medium filled with scatterers. We write the amplitudes of each spin eigenstate in a local mobile frame. One of the axes is in the direction of propagation of the particle. We use this representation to define a directional Green's operator of the homogeneous medium and also to write the spin-dependent scattering amplitudes. We show that this representation reveals a Berry phase. We establish a generalized Green-Dyson equation for the multiple-scattering problem in this framework. We show that the generalized Green-Dyson equation can be solved by linear algebra if one uses a representation of the rotations based on Wigner $D$ matrices. The properties of light scattering are retrieved if we use spin 1 particles. Our theory allows to take into account several kinds of anisotropies such as circular or linear dichroism and birefringence, Faraday effects, and Mie scattering within the same formalism. Several anisotropies can be present at the same time.
\end{abstract}

DOI: 10.1103/PhysRevE.80.056605

PACS number(s): 42.68.Mj, 78.20.Bh, 72.25.-b, 42.25.Ja

\section{INTRODUCTION}

In optics, several phenomena involve the polarization of light. Such phenomena, such as birefringence, dichroism, and Faraday effect, are well understood and described in textbooks [1], usually in the case where the medium is homogeneous and linear. When filled with particles, the medium is no longer homogeneous and, in addition to the transport of light, one has to study its scattering by the particles. A century ago, Mie discovered that the scattering of light by small dielectric spheres is linear and also affects the polarization $[2,3]$. The theory of light scattering has been extended for several different particles and studied in the context of multiple scattering [4-6]. While the polarization is well studied for a single scattering, the problem becomes more difficult in multiple scattering and a scalar approximation is often used. Such an approximation makes it simpler to investigate the coherent effects using sophisticated diagram techniques $[7,8]$. As a consequence, polarization effects are neglected whereas they may, in certain situations, be of interest. In magnetochiral birefringence, for instance, a residual degree of circular polarization persists in chiral media [9]. Recent experiments of coherent backscattering of light by cold atoms exhibit polarization-dependent enhancement factors [10]; this result is interpreted thanks to the quantum nonlinear nature of the cold atoms [11]. Under certain conditions, it was even forecast that an antilocalization effect could be observed [12]. Quantum effects of light were also introduced, in second quantization, for the study of dense cold atomic gases [13].

Remarkably, most of the scalar approximation techniques are applicable not only to light but also to microwaves, ultrasound, and noninteracting electrons, as emphasized in Ref. [7]. The existence of a polarization, alike, is not restricted to

\footnotetext{
*vincent.rossetto@grenoble.cnrs.fr
}

light; other waves display polarization effects: the other electromagnetic waves, elastic waves, and electrons. More generally, every particle with a spin is, from a quantum point of view, a polarized wave. The classical light polarization is a consequence of the vectorial nature of the electromagnetic field, whereas in the quantum-mechanical picture, photons have two states called helicities. The origin of the helicity of a particle follows from the spin. Relativity imposes that the spin 0 state of massless particles does not exist in the direction of propagation; photons therefore possess only two helicities. The freedom of the relative phase between the two helicity states is at the origin of the phenomenon of polarization as we experience it in optics. All quantum particles with a nonzero spin also have helicities and hence may display polarization effects. Therefore, the problem of polarization transport in multiple scattering does not only arise in optics. Polarization transport in multiple scattering is a more general problem and optics can be seen as the most investigated particular case.

According to the quantum duality of particles and waves, we may describe the propagation of particles as wave amplitudes from a statistical, or probabilistic, point of view. Reciprocally, the propagation of a wave can be described by the statistics of particle paths, through techniques such as Feynman's path integration. Following this picture, many theoretical calculations related to the multiple scattering of polarized waves are performed by Monte Carlo techniques. Such techniques have been developed for light, even in the presence of quantum scatterers [14], and for elastic waves [15]. Monte Carlo techniques are a pragmatic solution to the problem of taking into account each trajectory as a whole. Is it possible to consider each trajectory entirely and perform statistics in a more formal way? A pioneering work on trajectory statistics was made by Sato [16] with the introduction of a directional Green's function for the elastic energy (equivalent to the optical intensity) in a three-dimensional medium with nonisotropic scatterers. However, as his study con- 
cerned energy, polarization was not included in the model.

The trajectories of energy, or intensities, have been studied in a multiple-scattering context using Stokes parameters. These parameters are preferentially used by experimentalists because they are, as intensities, directly measurable quantities. They depend on the choice of the reference frame used for their observation. Their ability to take coherence effects into account is limited to intensity correlations. In order to dispose of the reference frame indetermination, Kuščer and Ribarič introduced harmonical functions to describe the Stokes parameters [17]. Based on Wigner's work concerning group theory [18], these functions have also been used under the name "spin-weighted spherical harmonics," though with a different phase factor [19]. They are also implicitly used in light-scattering studies under the name "vector spherical harmonics" [3,5]. The seminal work of Kuščer and Ribarič motivated a large number of studies on the phase matrix [20-22] and on the radiative transfer equation $[23,24]$.

The role of trajectories appears in a surprising effect involving polarized light. A four-foiled pattern is observed in backscattering experiments of polarized light from a medium containing anisotropic scatterers [25-27]. In these experiments, the source is not a planar infinite wave but a localized beam. The effect does not exist if the source is extended on a scale on the order of the four-foiled patterns. The same medium does not exhibit such patterns if the observations are made in transmission rather than in reflection, so that the properties of polarization depend not only on the detailed trajectory but also on the direction of observation, or more precisely on the frame of observation. It has been suggested that the appearance of these patterns is due to the existence of a Berry phase for the photon [28]. In quantum mechanics, Berry phases have been studied already in many situations $[29,30]$. In optics, the literature is abundant in experimental [31] and theoretical considerations [32,33]. If light is not guided in an optical fiber but rather multiply scattered, the Berry phase still exists [34]. The probability distribution of the phase depends on the statistics of the paths followed by the photons.

The purpose of the present paper is to introduce a general framework for the multiple scattering of any polarized wave, as simply and as pedagogically as possible. We will base our construction on the concept of path statistics, in the spirit of the Monte Carlo calculations, or Feynman's path integral, to account for local interactions and path-dependent effects. We express the formalism for particles with an arbitrary spin $S$, although we consider only linear media and scatterers and thus leave the other quantum properties to further investigations. Our objective is not to provide a complete theory for multiple scattering of polarized waves but rather to set up a framework in which such a theory could be developed. On that account, we assume the simplest possible point of view and make several assumptions, which are briefly discussed.

We have organized our work as follows. The representation of the field and the geometry of rotations are discussed in Sec. II. A directional Green's operator is discussed in Sec. III; it describes the transport in a homogeneous medium. Using the same formalism, we introduce the scattering operator between incoming and scattered state in Sec. IV. These first three sections constitute the elements of the theory. We demonstrate that our theory takes into account the Berry phase without requiring any extra tool in Sec. V. We derive a generalized Green-Dyson equation for multiple scattering of polarized waves in Sec. VI, and we show that a solution of this equation is obtained by means of the rotational harmonics introduced in Sec. VII. We discuss the particular case of rotational invariant scatterers in a rotational invariant medium in Sec. VIII and conclude. Fundamental formulas concerning the rotational harmonics are provided in Appendix $\mathrm{A}$, and some of the several notations used for them are listed in Appendix B.

\section{GEOMETRY AND POLARIZATION}

We begin the presentation of our work with the introduction of the geometry elements used throughout this paper. Mainly, the difference of our work relies on the representation of the state of waves in local frames. Polarization is introduced as a transformation property of the wave state under local rotations. We express all space coordinates in a fixed reference frame $X Y Z$. Any local frame $X^{\prime} Y^{\prime} Z^{\prime}$ is therefore obtained after a rotation of the reference frame. The coordinates of the local frame unit vectors, written in the reference frame, form a $3 \times 3$ matrix, which is the unique rotation matrix mapping $X Y Z$ into $X^{\prime} Y^{\prime} Z^{\prime}$ and preserving orientation. The group of rotations is $\mathrm{SO}(3)$. Considering the rotation associated to a frame is mathematically equivalent to considering the frame itself. We henceforth only use the description in terms of matrices for mathematical convenience and use exclusively the $Z Y Z$ Euler representation of rotation matrices, such that any rotation $R$ of $\mathrm{SO}(3)$ is decomposed into

$$
R=Z(\phi) Y(\theta) Z(\psi),
$$

where $Z(\phi)$ is the rotation around the $Z$ axis of angle $\phi$. Similarly, $Y$ is a rotation around the $Y$ axis. The decomposition (1) is unique if $0<\theta<\pi$ and $0 \leq \phi, \psi<2 \pi$. For $\theta=0$ or $\pi$, the decomposition is not unique.

In our discussion, we use the concept of trajectory or path of a particle. Studying the propagation of a wave using the particle picture would not be fully general if we were concerned by the behavior of single particles. But as our goal is to describe all possible trajectories as a whole, it is known that this way of addressing the problem of multiple scattering is correct. As a consequence, we will simultaneously use the wave picture, as equivalent to the statistics of particle trajectories, and the particle picture of the propagating wave. The spin of the particle emerges as an essential ingredient of the theory, after geometry considerations.

We study a medium filled with $N$ scatterers and write $\mathbf{r}_{i}$ for the position of the scatterer $i$. The trajectory of the particle is a succession of displacements between points $\mathbf{r}_{i}$ and changes in direction at the points $\mathbf{r}_{i}$. We call such a change in direction a scattering event. We consider the momentum $\mathbf{p}_{n}$ after $n$ scattering events and decompose it into

$$
\mathbf{p}_{n}=p_{n} P_{n} \hat{\mathbf{z}}
$$

where $p_{n}=\left|\mathbf{p}_{n}\right|$ is the modulus of $\mathbf{p}_{n}, P_{n}$ is a rotation matrix (expressed in the reference frame), and $\hat{\mathbf{z}}$ is the unit vector of 
the reference frame along the third coordinate. We choose the local frame for the segment of the path after the $n$th scattering event and before the next one. The third coordinate of the local frame is therefore always pointing in the direction of motion.

We remark immediately that the decomposition (2) of $\mathbf{p}_{n}$ is not unique. The Euler angle $\psi$ of $P_{n}$ can be taken arbitrarily; it is the spin gauge freedom. Here stands a crucial point in our model. The introduction of the local frame is a natural way of introducing the spin and, hence, the polarization, into a multiple-scattering formulation. If the wave has a spin $S$, there are $2 S+1$ possible values for the spin component along the direction of propagation that we label with " $s$ " $(-S \leq s \leq S)$. At a given point $\mathbf{r}$, the field is a superposition of partial fields, which we consider to be plane waves, with different momenta. For simplicity, we may consider all directions of propagation for a fixed absolute value of the momentum. The amplitude of probability for observing the spin state $s$ in the frame $P$ at point $\mathbf{r}$ and time $t$ is written $\Psi(\mathbf{r}, t, P, s)$. In this paper, we regularly use the bracket notation for functions. The functions $f$ of a variable $x$ are written as $\langle x \mid f\rangle$, instead of $f(x)$. There is no consequence of this notation concerning the physics itself, but we find it more convenient for our purpose, in particular, for the introduction of the rotational harmonics in Sec. VI. We then write the amplitude as a bracket product

$$
\Psi(\mathbf{r}, t, P, s)=\langle\mathbf{r}, P, s \mid \Psi(t)\rangle .
$$

Polarization is related to the frame of observation and if this frame is changed, polarization is modified according to certain rules. Light polarization, for instance, is turned by an angle $-\psi$ if the frame of observation is turned by an angle $\psi$ around the direction of propagation. It is therefore natural to change the basis of representation and use the eigenbasis of rotation along the direction of propagation. Light has two circular polarization states, corresponding to the helicities of the photon. A rotation of angle $\psi$ creates a phase shift for each circular eigenstate. If $s$ is an eigenvalue of the spin operator along the direction of propagation, rotations along this axis commute with the spin operator. Unitarity implies that

$$
\langle P Z(\psi), s|=e^{\mathrm{i} s \psi}\langle P, s|
$$

on the on-shell states. After these remarks, we shall name the third Euler angle, usually noted $\psi$, the spin angle.

We have presented a description of the field at a given point that depends on the local frame and, thereby, taking into account the spin. We still have to check the completeness of the ket representation $|P, s\rangle$. As we have remarked, the direction of propagation is $P \hat{\mathbf{z}}$, so that $|P, s\rangle$ and $\left|P^{\prime}, s^{\prime}\right\rangle$ are orthogonal if $P \hat{\mathbf{z}} \neq P^{\prime} \hat{\mathbf{z}}$. From relation (1), we conclude that nonorthogonal $|P, s\rangle$ and $\left|P^{\prime}, s^{\prime}\right\rangle$ have the same Euler angles except the spin angle, which is arbitrary. Let $R$ and $R^{\prime}$ be two reference frames; we introduce the function $\delta_{\perp}\left(R^{-1} R^{\prime}\right)$ to denote

$$
\delta_{\perp}\left(R^{-1} R^{\prime}\right) \equiv 4 \pi \delta\left(\cos \theta^{\prime}-\cos \theta\right) \delta\left(\phi^{\prime}-\phi\right),
$$

where $\theta$ and $\phi$ are the first two Euler angles of $R$ and $\theta^{\prime}$ and $\phi^{\prime}$ the first two Euler angles of $R^{\prime} . \delta_{\perp}$ is a kind of Dirac function for rotation matrices, which select frames having the same third unit vector. Using this notation, the condition $P \hat{\mathbf{z}}=P^{\prime} \hat{\mathbf{z}}$ is imposed by defining $\left\langle P^{\prime}, s^{\prime} \mid P, s\right\rangle$ proportional to $\delta_{\perp}\left(P^{-1} P^{\prime}\right)$. We remark that $\delta_{\perp}\left(P^{\prime-1} P\right)=\delta_{\perp}\left(P^{-1} P^{\prime}\right)$. Finally, the orthogonality of the spin eigenstates yields $\left\langle s \mid s^{\prime}\right\rangle=\delta_{s s^{\prime}}$. From the expression (4), we get the product

$$
\left\langle P^{\prime}, s^{\prime} \mid P, s\right\rangle=e^{\mathrm{i} s^{\prime} \psi^{\prime}-\mathrm{i} s \psi} \delta_{\perp}\left(P^{-1} P^{\prime}\right) \delta_{s s^{\prime}}
$$

where $\psi$ and $\psi^{\prime}$ are the spin angles of $P$ and $P^{\prime}$, respectively.

The superposition of the partial fields for all directions of propagations is expressed by

$$
\begin{aligned}
\int_{\mathrm{SO}(3)} & d P \Psi(\mathbf{r}, t, P, s) e^{-\mathrm{i} s \psi} \\
= & \frac{1}{8 \pi^{2}} \int_{0}^{\pi} \sin \theta d \theta \int_{0}^{2 \pi} d \phi \int_{0}^{2 \pi} d \psi \\
& \times \Psi[\mathbf{r}, t, Z(\phi) Y(\theta) Z(\psi), s] e^{-\mathrm{i} s \psi} \\
= & \frac{1}{4 \pi} \int_{0}^{\pi} \sin \theta d \theta \int_{0}^{2 \pi} d \phi \Psi[\mathbf{r}, t, Z(\phi) Y(\theta), s] \equiv \Psi_{s}(\mathbf{r}, t)
\end{aligned}
$$

The presence of the term $e^{\mathrm{is} \psi}$ is essential. Its role is to add the amplitudes in such a way that the amplitudes in two distinct frames with the same direction of propagation are in phase and do not cancel out. Forgetting this term would make all $\Psi_{s}$ vanish except $\Psi_{0}$, because the exponential term from Eq. (4) would be integrated to give 0. Multiplescattering theories for scalar waves actually only consider the term $\Psi_{0}$. The completeness of the Hilbert basis $|P, s\rangle$ is expressed by the closure formula ( $\hat{1}$ is the identity operator)

$$
\sum_{s=-S}^{S} \int_{\mathrm{SO}(3)} d P|P, s\rangle\langle P, s|=\hat{1} .
$$

The integral over $\mathrm{SO}(3)$ has been defined in Eq. (7). The description we have introduced in this section will be used in the formulation of the multiple scattering for polarized waves. In the next section, we introduce the Green's operator for the polarized states $|P, s\rangle$ and its space dependence in the absence of scatterers.

\section{TRANSPORT IN A HOMOGENEOUS MEDIUM}

Before studying multiple scattering, it is necessary to investigate the transport in a homogeneous medium. By the word transport, we mean the response at a position $\mathbf{r}^{\prime}$ and time $t^{\prime}$ in the frame $P^{\prime}$ (see previous section for the introduction of the frames) to a source at position $\mathbf{r}$ and time $t$ in the frame $P$. It is advantageous to use the Green's functions because the two situations of massive and massless particles can both be handled with the same formalism. In the case of a massless particle, such as the photon, transport follows from Maxwell's equations and is often formulated by the Helmholtz equation. Massive particles transport is of a different nature and the dynamics of their wave function responds to the Schrödinger equation. 
We denote by $\mathcal{G}$ the Green's function of the operator $\frac{\partial^{2}}{\partial r^{2}}$ $+\frac{\omega^{2}}{c^{2}}$ or of the operator $-\frac{\hbar^{2}}{2 m} \frac{\partial^{2}}{\partial r^{2}}-E$ to describe the transport of the electromagnetic field or of the wave function, respectively. The equivalence between these two operators is obtained through the relation

$$
\frac{\omega}{c}=\frac{1}{\hbar} \sqrt{2 m E},
$$

so that we shall use in our formulas only the "massless" notation $\omega / c$. The results we present can be extended to massive particles of arbitrary spin by using Eq. (9) and changing the sign of $\mathcal{G}$. If the medium is invariant under translation and in time $\mathcal{G}\left(\mathbf{r}, t ; \mathbf{r}^{\prime}, t^{\prime}\right)$ depends on $\mathbf{r}^{\prime}-\mathbf{r}$ and $t^{\prime}-t$. In this section, we construct a Green's operator $\hat{G}_{0}$, which depends on the direction of motion at $\mathbf{r}$ and $\mathbf{r}^{\prime}$, and we call it the directional free Green's operator.

We introduce the free Green's operator as the operator transforming the wave function along the propagation of the wave if no scattering events occur. The transition amplitudes characterizing the response may depend on the spin. If we note $\hat{G}_{0}$ the free Green's operator, transport is described by the matrix elements $\left\langle\mathbf{r}^{\prime}, P^{\prime}, s^{\prime}\left|\hat{G}_{0}\left(t^{\prime}, t\right)\right| \mathbf{r}, P, s\right\rangle$. We have introduced the position ket $|\mathbf{r}\rangle$ and denoted $|\mathbf{r}, P, s\rangle \equiv|\mathbf{r}\rangle$ $\otimes|P, s\rangle$. Naturally, it is not physical in quantum mechanics to consider the position and the direction of motion of a particle simultaneously. The directional Green's operator that we need for our theory can be seen as an intermediate element of computation. The physical Green's function is the superposition of the directional Green's operators for all initial and all final directions of motion. In Feynman's picture of path integrals, it corresponds simply to decompose the pathintegral formulation of $\mathcal{G}$ into path integrals over trajectories with a constraint on the direction of motion at the initial and final points. We have illustrated this feature in Fig. 1.

In a medium with translational invariance, the momentum is conserved so that $\hat{G}_{0}$ has a factor $\left\langle P \mid P^{\prime}\right\rangle$. Between two scattering events, the wave travels in space from $\mathbf{r}$ to $\mathbf{r}^{\prime}$ along the direction $\hat{\mathbf{p}}=P \hat{\mathbf{z}}$. The direction of the momentum $P \hat{\mathbf{z}}$ is the same as the direction $\mathbf{r}^{\prime}-\mathbf{r}$ because of the relation $\mathbf{r}^{\prime}-\mathbf{r}=c\left(t^{\prime}-t\right) P \hat{\mathbf{z}}$, so that we have to impose this constraint to the free Green's operator. To express the directional constraint on $\mathbf{r}^{\prime}-\mathbf{r}$, we write a similar equation as Eq. (2),

$$
\mathbf{r}^{\prime}-\mathbf{r}=r D \hat{\mathbf{z}}
$$

so that the dependence of the Green's operator $\hat{G}_{0}$ on $D$ is simply reduced to $\delta_{\perp}\left(D P^{-1}\right)$. Finally, we have the expression for the dynamics Green's operator

$$
\begin{aligned}
& \left\langle\mathbf{r}^{\prime}, P^{\prime}, s^{\prime}\left|\hat{G}_{\mathrm{dyn}}\left(t^{\prime}, t\right)\right| \mathbf{r}, P, s\right\rangle \\
& \quad=\mathcal{G}\left(\mathbf{r}, t ; \mathbf{r}^{\prime}, t^{\prime}\right) \delta_{\perp}\left(D P^{-1}\right)\left\langle P^{\prime}, s^{\prime} \mid P, s\right\rangle .
\end{aligned}
$$

The factor $\delta_{\perp}\left(D P^{-1}\right)$ in expression (11) is a very strong restriction imposed by rotational invariance. It states that a rotation of the medium must be accompanied by the same rotation of the frames $P$ and $P^{\prime}$ (through the term $\left.\left\langle P^{\prime}, s^{\prime} \mid P, s\right\rangle\right)$ to leave the Green's operator unchanged.
In the presence of scatterers, it has been observed in backscattering configurations that the transport of polarization depends on the relative directions of the incident polarization beam and the vector $\mathbf{r}^{\prime}-\mathbf{r}$ [26]. Moreover, in some cases, such as in the presence of linear birefringence or dichroism, the direction of propagation has a strong influence on the transport. Such complex geometrical dependences will be described by the dependence on $P, P^{\prime}$, and $D$ of the generalized Green's operator.

We now consider the evolution of the spin amplitudes during the transport. In the simple case, where the time evolution of the spin does not depend on position or time, it is described by

$$
\hat{G}_{\text {spin }}\left(t^{\prime}, t\right)=\exp \left[-\frac{i}{\hbar} \hat{A}_{\text {spin }}\left(t^{\prime}-t\right)\right],
$$

where $\hat{A}_{\text {spin }}$ is the evolution operator of the spin. If there is no anisotropy associated with the spin and if the spin is conserved during transport, then the terms $\left\langle s^{\prime}\left|\hat{G}_{\text {spin }}\right| s\right\rangle$ form a $(2 S+1) \times(2 S+1)$ diagonal unitary matrix. Effects depending on the spin, such as circular birefringence or dichroism, modify the diagonal terms of $\left\langle s^{\prime}\left|\hat{G}_{\text {spin }}\right| s\right\rangle$ such that it is not necessarily unitary anymore. Consider, for instance, a medium with absorption length $\kappa_{s}^{-1}$ and index $n_{s}$ for the spin $s$. In this case, we have

$$
\begin{aligned}
& \left\langle\mathbf{r}^{\prime}, P^{\prime}, s^{\prime}\left|\hat{G}_{\text {spin }}\left(t^{\prime}, t\right)\right| \mathbf{r}, P, s\right\rangle \\
& \quad=e^{-\mathrm{i}\left(n_{s}\left(\omega-\mathrm{i} c \kappa_{s}\right)\left(t^{\prime}-t\right)\right.} \delta^{(3)}\left(\mathbf{r}^{\prime}-\mathbf{r}\right)\left\langle P^{\prime}, s^{\prime} \mid P, s\right\rangle .
\end{aligned}
$$

In this expression, the index $n_{s}$ denotes the ratio of wave celerity for a spin eigenstate $s$ compared to the celerity contained in $\mathcal{G}$. Dichroism appears when the values of $\kappa_{s}$ depend on $s$, birefringence when the values of $n_{s}$ depend on $s$. It is also possible to introduce spin flips.

To summarize our construction of the Green's operator of the homogeneous medium, we have the following formula for $\hat{G}_{0}$ :

$$
\begin{aligned}
&\left\langle\mathbf{r}^{\prime}, P^{\prime}, s^{\prime}\left|\hat{G}_{0}\left(t^{\prime}, t\right)\right| \mathbf{r}, P, s\right\rangle \\
&= \mathcal{G}\left(\mathbf{r}^{\prime}-\mathbf{r}, t^{\prime}-t\right)\left\langle s^{\prime}\left|\hat{G}_{\text {spin }}\left(t^{\prime}, t\right)\right| s\right\rangle \\
& \quad \times \delta_{\perp}\left(D P^{-1}\right) \delta_{\perp}\left(P^{-1} P\right) e^{\mathrm{i}\left(s^{\prime} \psi^{\prime}-s \psi\right)} .
\end{aligned}
$$

The dependence of the Green's function as a function of the distance is, according to the expression (14), the product of the scalar Green's function $\mathcal{G}$ and the Green's function of the spin $\hat{G}_{\text {spin. }}$. If there is no absorption, the Green's function follows the well-known $r^{-1}$ decrease in the Green's function in three dimensions. In this situation, the enhanced Green's function (14) verifies the conservation of energy by construction.

\section{DESCRIPTION OF A SCATTERING EVENT}

In the previous section, we have constructed the Green's function for a homogeneous medium. In this section, we include the description of the scatterers into the same formal- 


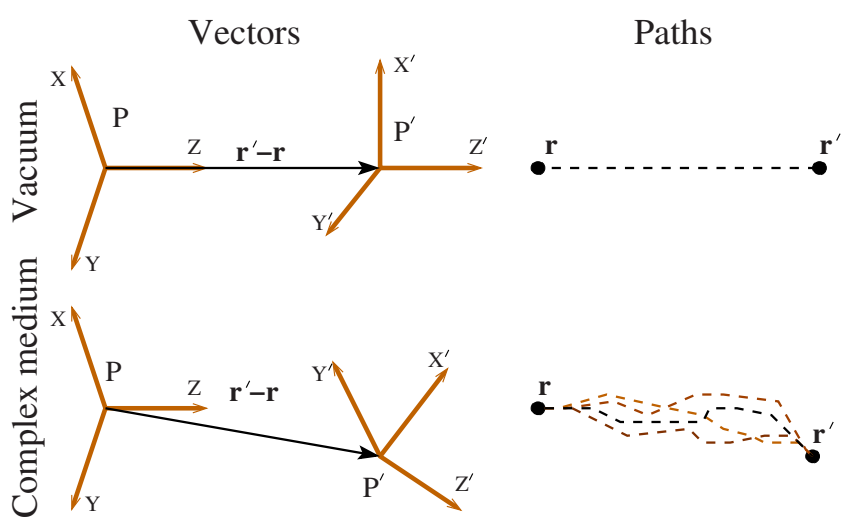

FIG. 1. (Color online) Schematic view of the directional Green's operator. Without scatterers (upper figures), the only situation for which the Green's operator does not vanish is when the three vectors $\mathbf{r}^{\prime}-\mathbf{r}, P \hat{\mathbf{z}}$, and $P^{\prime} \hat{\mathbf{z}}$ are colinear and have the same direction (left-hand side of the figure). In this situation, only one path is possible: the straight line from $\mathbf{r}$ to $\mathbf{r}^{\prime}$ sketched as a dashed line on the right-hand side of the figure. In a complex medium (lower figures), the directional Green's operator $\left\langle\mathbf{r}^{\prime}, P^{\prime}\left|\hat{G}_{0}\right| \mathbf{r}, P\right\rangle$ describes the transport from $\mathbf{r}$ to $\mathbf{r}^{\prime}$ with the constraint that the initial and final directions of motion are $P \hat{\mathbf{z}}$ and $P^{\prime} \hat{\mathbf{z}}$, respectively. There are several different paths, some examples of which are shown as dashed lines on the right-hand side in the figure. Note that the vectors $\mathbf{r}^{\prime}-\mathbf{r}, P \hat{\mathbf{z}}$, and $P^{\prime} \hat{\mathbf{z}}$ need not to be colinear anymore.

ism. The problem of scattering with spin particles was originally discussed by Jacob and Wick [35] when two particles with spins are colliding. We consider more general scatterers in our formalism and describe an arbitrary interaction in the far field. In this situation, we only need to know the so-called on-shell " $T$ matrix," or transition matrix, in a theory of multiple scattering.

We use the following definition of the on-shell $T$ matrix, in the case of light scattering. Consider an incoming field $\mathbf{E}_{\text {in }}$ and a scatterer at position $\mathbf{r}$. At a far-field position, the scattered field $\mathbf{E}_{\text {sca }}$ depends linearly on $\mathbf{E}_{\text {in }}$,

$$
\mathbf{E}_{\mathrm{sca}}\left(\mathbf{r}^{\prime}, t^{\prime}\right)=\mathcal{G}\left(\mathbf{r}^{\prime}, t^{\prime} ; \mathbf{r}, t\right) T \mathbf{E}_{\mathrm{in}}(\mathbf{r}, t)
$$

The $T$ matrix can be computed or measured in several ways that we will not discuss. In general, the $T$ matrix depends on the incoming and outgoing directions and on the polariza-

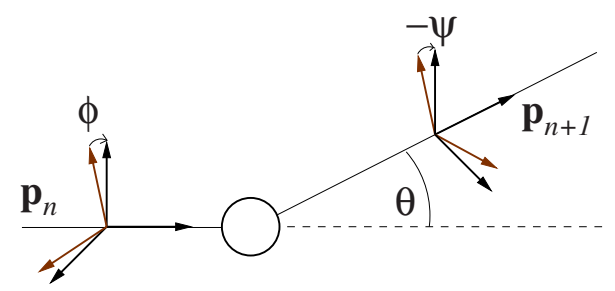

FIG. 2. (Color online) A single-scattering event. The freedom of rotation of the incoming and outgoing frames is compensated by a local rotation of the spin angle (4). The azimuth angle $\widetilde{\phi}$ of $P_{n}^{-1} P_{n+1}$ determines the scattering plane and the zenith angle $\tilde{\theta}$ of $P_{n}^{-1} P_{n+1}$ is the scattering angle. tion. These dependences will be rigorously taken into account in the present formalism.

The position in space of the scatterers are denoted by $\mathbf{r}_{i}$. A scattering event corresponds to a change in the direction of propagation at $\mathbf{r}_{i}$ from $P_{n} \hat{\mathbf{z}}$ to $P_{n+1} \hat{\mathbf{z}}$. In the laboratory frame, the rotation matrix corresponding to a scattering event is $R_{0}=P_{n+1} P_{n}^{-1}$. However, it is the local rotation, which is physically relevant in a local description of scattering. The expression of this local rotation is $R=P_{n}^{-1} R_{0} P_{n}=P_{n}^{-1} P_{n+1}$. This is the local rotation experienced from the point of view of the particle during the scattering event. Using the decomposition of $R$ into Euler angles $(\widetilde{\phi}, \widetilde{\theta}, \widetilde{\psi})$ [Eq. (1)], we rewrite $P_{n+1}=P_{n} R$ as

$$
P_{n+1} Z(\widetilde{\psi})^{-1}=P_{n} Z(\widetilde{\phi}) Y(\tilde{\theta}) .
$$

$\tilde{\theta}$ is the scattering angle and $\widetilde{\phi}$ and $-\widetilde{\psi}$ will act as spin angles in Eq. (4). As a consequence of this relation, the modifications of the spin angles of $P_{n}$ and $P_{n+1}$ transform according to

$$
\begin{gathered}
P_{n} \rightarrow P_{n} Z(\widetilde{\phi}), \\
P_{n+1} \rightarrow P_{n+1} Z(-\tilde{\psi}),
\end{gathered}
$$

and cast the rotation into local frames adapted to the scattering event (see Fig. 2).

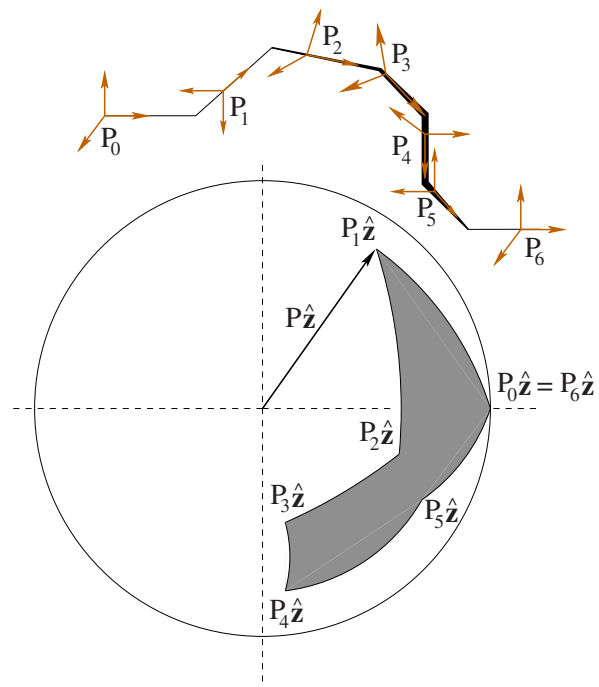

FIG. 3. (Color online) Example in the case $n=6$ of the Berry phase for a trajectory with identical initial and final frames. (Top) Trajectory in real space. The thickness of the line changes along the trajectory to give a three-dimensional effect: the closer it is to the observer, the thicker the line is. On each segment is represented a frame $P_{i}$ such that $P_{i} \hat{\mathbf{z}}$ is the direction of the segment. (Bottom) The path is represented on the unit sphere. Each segment with constant direction $P_{i} \hat{\mathbf{z}}$ is a point on the sphere. Two successive points are connected by an arc of a circle. The area enclosed by the path on the sphere is the geometric phase $\Omega$ (in gray). The angles $\tilde{\theta}_{i}$ (see text) have been chosen exaggeratedly large for the readability of both parts of the figure. In strong forward scattering, the trajectory on the top part is very close to a straight line and the area enclosed on the sphere of the bottom part is concentrated around $P_{0} \hat{\mathbf{z}}$. 
If there is a spherical scatterer at position $\mathbf{r}$, the scattering operator between incoming and outgoing states is

$$
\begin{aligned}
& \left\langle\mathbf{r}^{\prime}, P_{n+1}, s^{\prime}\left|\hat{T}\left(t^{\prime}, t\right)\right| \mathbf{r}, P_{n}, s\right\rangle \\
& \quad=\delta^{(3)}\left(\mathbf{r}^{\prime}-\mathbf{r}\right) \delta\left(t^{\prime}-t\right) e^{\mathrm{i} s \tilde{\phi}+\mathrm{is} s^{\prime}} \tilde{\psi}\left\langle Y(\widetilde{\theta}), s^{\prime}|\hat{T}| \mathrm{I}, s\right\rangle
\end{aligned}
$$

( $Y$ is a rotation around the $Y$ axis of the reference frame). We have used the spherical symmetry of the scatterer to bring the incoming and outgoing frames back into the incoming one, so that the outgoing frame is simply $Y(\widetilde{\theta})$. In this formula- tion, $\left\langle Y(\tilde{\theta}), s^{\prime}|\hat{T}| I, s\right\rangle$ is just the usual $T$ matrix written in the spin eigenstates basis, which we write $\mathcal{T}_{s s^{\prime}}(\omega, \tilde{\theta})$. We impose that there is a scatterer at position $\mathbf{r}$ with the density operator $\left\langle\mathbf{r}^{\prime}|\hat{\varrho}| \mathbf{r}\right\rangle=\delta^{(3)}\left(\mathbf{r}^{\prime}-\mathbf{r}\right) \varrho(\mathbf{r})$, with

$$
\varrho(\mathbf{r})=\sum_{i=1}^{N} \delta^{(3)}\left(\mathbf{r}-\mathbf{r}_{i}\right)
$$

If there is no scatterer at position $\mathbf{r}$, the scattered field vanishes. The scattered field is obtained using the closure relation (8)

$$
\begin{aligned}
\left\langle\mathbf{r}^{\prime}, P^{\prime}, s^{\prime}\left|\hat{G}_{0}\left(t^{\prime}, t\right) \hat{T} \hat{\varrho}\right| \mathbf{r}, P, s\right\rangle & =\int_{\mathcal{V}} d^{3} \mathbf{r}^{\prime \prime} \int_{\mathrm{SO}(3)} d P^{\prime \prime} \sum_{s^{\prime \prime}=-S}^{S}\left\langle\mathbf{r}^{\prime}, P^{\prime}, s^{\prime}\left|\hat{G}_{0}(t, t)\right| \mathbf{r}^{\prime \prime}, P^{\prime \prime}, s^{\prime \prime}\right\rangle\left\langle\mathbf{r}^{\prime \prime}, P^{\prime \prime}, s^{\prime \prime}|\hat{T} \varrho| \mathbf{r}, P, s\right\rangle \\
& =\mathcal{G}\left(\mathbf{r}^{\prime}-\mathbf{r}, t^{\prime}-t\right) \delta_{\perp}\left(D P^{\prime-1}\right) \varrho(\mathbf{r}) \sum_{s^{\prime \prime}=-S}^{S}\left\langle s^{\prime}\left|\hat{G}_{\mathrm{spin}}\left(t^{\prime}-t\right)\right| s^{\prime \prime}\right\rangle e^{\mathrm{i}\left(s^{\prime}-s^{\prime \prime}\right) \psi^{\prime}} e^{\mathrm{i} s \tilde{\phi}+\mathrm{is} s^{\prime \prime}} \tilde{\mathcal{T}}_{s s^{\prime \prime}}(\omega, \widetilde{\theta}) .
\end{aligned}
$$

To obtain the last equality, we have used the fact that the spin angle of the rotation matrix $P^{\prime \prime}$ is arbitrary and does not play any role in the final expression and we have therefore used the spin angle of $P^{\prime}$ to simplify the formula. The angles $(\widetilde{\phi}, \widetilde{\theta}, \widetilde{\psi})$ are the Euler angles of $P^{-1} P^{\prime}$.

The $\hat{T}$ operator in formula (21) stands for a single spherical scatterer, correlations or the influence of the other scatterers are, so far, ignored. The imaginary part of $\hat{T}$ stands for extinction. The extinction cross section for spin $s$ is then

$$
\sigma_{\text {ext }}^{s}=-\frac{c}{\omega} \operatorname{Im} \mathcal{T}_{s s}(\omega, \theta=0) .
$$

It is constructive to compare $\sigma_{\text {ext }}$ with the scattering cross section for spin $s$

$$
\sigma_{\text {scatt }}^{s}=\frac{1}{2} \sum_{s^{\prime}=-S}^{S} \int_{0}^{\pi} \sin \theta d \theta\left|\mathcal{T}_{s s^{\prime}}(\omega, \theta)\right|^{2} .
$$

The total extinction and scattering cross sections are obtained by summing over the spin eigenstates $\sigma_{\mathrm{ext}}=\Sigma_{s} \sigma_{\mathrm{ext}}^{s}$ and $\sigma_{\text {scatt }}=\sum_{s} \sigma_{\text {scatt. }}^{s}$. By conservation of energy, the ratio $a$ $=\sigma_{\text {scatt }} / \sigma_{\text {ext }}$ cannot exceed 1 . The ratio $a$ is called the albedo. If $\sigma_{\text {ext }}=\sigma_{\text {scatt }}$, all energy captured by the particle is scattered. This is known as the optical theorem. Let us remark that the inequality $a \leq 1$ valid for the total albedo is not necessarily true for the albedoes $a^{s}=\sigma_{\text {scatt }}^{s} / \sigma_{\text {ext }}^{s}$ at fixed spin.

\section{BERRY PHASE}

At this point of the discussion, it is interesting to point out that our description of the trajectory is able to keep track of the Berry phase of the wave. Originally, the Berry phase was proposed as the phase factor that can appear after a cycle during an adiabatic time evolution of a nondegenerated quantum state [29]. Later, it was discovered that the concept applies to light polarization as well $[31,36]$ and that the time evolution of the system does not need to be cyclic or unitary [37]. More recently, it was shown that the Berry phase also shows up in multiply scattered light [34]. The expression for the Berry phase $\Phi$ involves two factors: the spin $s$ of the particle and a solid angle $\Omega$ (which we call the geometric phase) or, more generally, the curvature of the phase space of the system enclosed by the evolution of the system during one cycle. The Berry phase expression is [29]

$$
\Phi=-s \Omega .
$$

In polymer physics, time is replaced by a curvilinear space coordinate and the geometric phase $\Omega$ is called writhe [38]. The fluctuations of the writhe induced by thermal fluctuations of the polymer's shape have been studied numerically using a Monte Carlo approach [39]. For long polymers, the distribution of the Berry phase was found Gaussian. There is no exact approach for the statistics of the Berry phase for random paths.

For simplicity, we consider the equivalent of a nearly adiabatic time evolution and compare the phases of the field before and after a path for which the initial and final frames are equal. Such a path corresponds to a closed circuit in the phase space [31]. We also assume that the scattering is elastic and that the spin is conserved in both scattering and propagation. Between two scattering events, the state of a particle is simply defined by the direction of its momentum, and the phase space is thus the unit sphere. The adiabatic evolution would correspond to a continuous and slow movement of the direction of the momentum on the sphere. In case of strong forward scattering, each scattering event corresponds to a small change in the direction of propagation, which nearly satisfies the adiabatic condition for the Berry phase to occur. 
The geometric phase is the solid angle enclosed by the unit wave vector $\hat{\mathbf{p}}=P \hat{\mathbf{z}}$ along its trajectory on the unit sphere.

To reveal the Berry phase, we consider a trajectory made of $n$ scattering events and the $n+1$ local frames associated to each propagation. We denote by $\left(\phi_{i}, \theta_{i}, \psi_{i}\right)$ the Euler angles of each frame after the decomposition of Eq. (2). An example of this construction, with $n=6$, is shown on Fig. 3. We consider the case of strong forward scattering so that the coordinates $\left(\theta_{i+1}, \phi_{i+1}\right)$ and $\left(\theta_{i}, \phi_{i}\right)$ of the frames $P_{i+1}$ and $P_{i}$ are close to one another. In Fig. 3, it corresponds to the case, where the points $P_{i} \hat{\mathbf{z}}$ and $P_{i+1} \hat{\mathbf{z}}$ on the unit sphere are close to each other, compared to the radius of the sphere. We call $\left(\widetilde{\phi}_{i}, \widetilde{\theta}_{i}, \widetilde{\psi}_{i}\right)$ the Euler angles of the rotations $P_{i}^{-1} P_{i+1}$ in the same way as we have done in Eq. (16). The strong scattering assumption corresponds to $\tilde{\theta}_{i} \ll 1$. To ensure readability of Fig. 3, we have represented a path with large angles $\tilde{\theta}_{i}$ that do not correspond to a strong forward scattering. By analogy with a spinning top, for which the instantaneous rotation around the third axis is expressed by $\dot{\phi} \cos \theta+\dot{\psi}$ [40], Chap. VI, we deduce that the rotation around its direction of propagation experienced by the particle is $\widetilde{\phi}_{i}+\widetilde{\psi}_{i} \simeq\left(\phi_{i+1}\right.$ $\left.-\phi_{i}\right) \cos \theta_{i}+\psi_{i+1}-\psi_{i}$. The Berry phase for a given component of the field is, in our case, identified as the phase difference between the values of this component expressed in the two frames $P_{0}$ and $P_{n}$. The original definition of the Berry phase demands that the evolution be cyclic. So we have to impose that the frames $P_{0}$ and $P_{n}$ are equal, which corresponds to one cycle. Therefore, we have $\phi_{n}=\phi_{0} \bmod 2 \pi$ and $\psi_{n}$ $=\psi_{0} \bmod 2 \pi$. The extra phase for a spin state $s$ between the frames 0 and $n$ is, according to the phase factor $e^{\mathrm{i}\left(s \tilde{\psi}+s^{\prime} \tilde{\phi}\right)}$ in Eq. (21),

$$
\Phi=s \sum_{i=0}^{n-1} \widetilde{\phi}_{i}+\widetilde{\psi}_{i} .
$$

We have used the conservation of the spin $\left(s^{\prime}=s\right)$. We rewrite the sum using the quantities $\left(1-\cos \theta_{i}\right)$ and we get, up to a multiple of $2 \pi$,

$$
\Phi=-s \sum_{i=0}^{n-1}\left(1-\cos \theta_{i}\right)\left(\phi_{i+1}-\phi_{i}\right)=-s \Omega \bmod 2 \pi .
$$

We recognize that the sum in Eq. (26) is a spherical area (Fig. 3). It is equal to the geometric phase up to a multiple of $4 \pi$ because of the indetermination of the solid angle [39]. We have retrieved the expression of the Berry phase (24). The equality between the sum in expression (26) and the geometric phase holds modulo $4 \pi$, rather than $2 \pi$, for topological reasons [28]. The indetermination modulo $4 \pi$ plays here no role because $s$ is an integer or a half-integer, so the phase is undetermined modulo $2 \pi$.

Remarkably, the Berry phase emerges in our local frame model without any special effort. The geometrical nature of the Berry phase appears here clearly. As it was already demonstrated, the existence of the Berry phase is not restricted to cyclic systems and can be extended to nonclose path and to nonunitary evolution (in our theory, if there is absorption, for instance) [37]. The statistics of the Berry phase for random
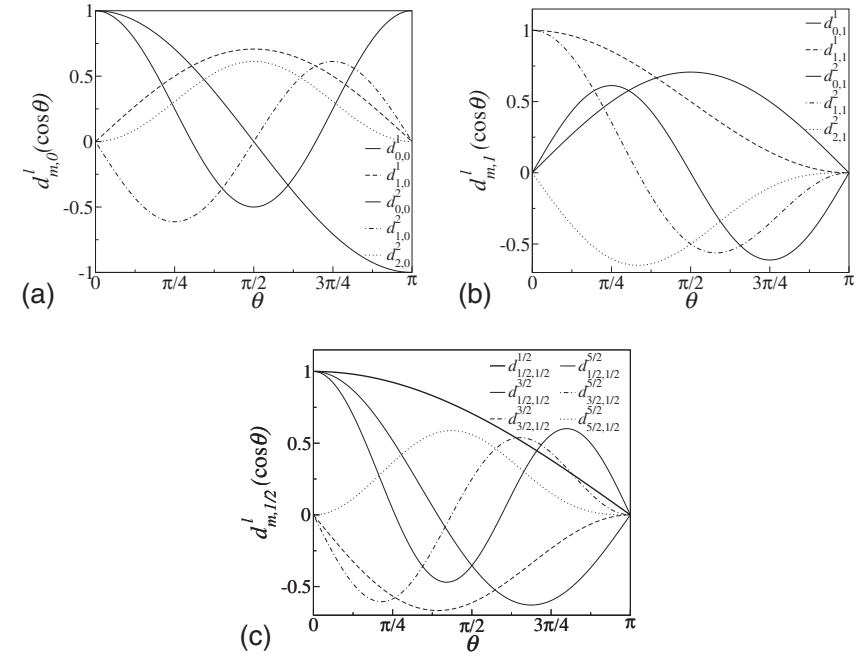

FIG. 4. Examples of functions $d_{m n}^{l}(\cos \theta)$ for small values of $l$. The only function with $l=0, d_{00}^{0}=1$ is not shown. Top: graphs of functions with spin index 0 for $l=1$ and $l=2$. One recognizes the unnormalized spherical harmonics. Middle: graphs of functions with spin index 1 for $l=1$ and $l=2$. Bottom: graphs of functions with spin index $1 / 2$ for $l=1 / 2$ and $l=3 / 2$.

walks is a technical and difficult problem [41,42]. Our approach will make it possible to obtain exact results concerning the Berry phase statistics.

A possible experiment to observe the Berry phase of light consists in setting a directional linearly polarized source at the edge of a slab of a medium filled with scatterers and observe the outgoing light through a collimator and a linear polarizer. To ensure strong forward scattering, the scatterers must be large such that the anisotropy $g$ defined as the average cosine of the scattering angle verifies $1-g \ll 1$. One can, for instance, place the polarizer in front of the source, its plane orthogonal to the direct beam and its axis making an angle $\theta$ with the polarization of the source. The intensity as a function of $\theta$ will display deviations from the expected $\cos ^{2} \theta$ behavior if there were no scatterers. If the slab depth is large, the Berry phase will spread out on a width larger than $2 \pi$ and the intensity will be constant as a function of $\theta$. The experiment has therefore to be performed with slab depths of a few transport mean free paths $\ell^{*}=\ell /(1-g)(\ell$ is defined in the next section). Different concentrations will lead to different deviations.

\section{GENERALIZED GREEN-DYSON EQUATION}

The complexity in multiple scattering stems from the summation of the contributions to the field of all the paths from the source to the observer and potentially from their interferences. In a first approximation, one often neglects these interferences and assumes that the scattering events are independent from each other. In a disordered system, the averaging over disorder can thus be performed without the sophisticated diagram techniques needed to preserve interference effects. The elements of the theory that we have introduced in Secs. III and IV are used in this section to write a generalized Green-Dyson equation for the multiple scattering 
of polarized waves under the assumption of independent scattering events. This equation applies to the directional Green's operators. It relates the free Green's operator $\hat{G}_{0}$ we have introduced in Sec. III to the effective Green's operator $\hat{G}$ of the same medium filled with scatterers. In the second part of this section, we introduce the Fourier transform of the generalized Green-Dyson equation.

Consider a medium filled with scatterers at fixed positions $\mathbf{r}_{i}$. The presence of the scatterers is equivalent to the introduction of the perturbation in the transport equation. In the formalism of the Green's functions, it corresponds to introducing a transition matrix or $T$ matrix. We now consider the perturbed Green's operator $\hat{G}$ developed as a Born expansion in terms of $\hat{G}_{0}$,

$$
\hat{G}=\hat{G}_{0}+\hat{G}_{0} \hat{T} \hat{\varrho} \hat{G}_{0}+\hat{G}_{0} \hat{T} \hat{\varrho} \hat{G}_{0} \hat{T} \hat{\varrho} \hat{G}_{0}+\cdots .
$$

The first term of the right-hand side of Eq. (27) stands for the unscattered field, the second term for the single scattering, and so on. Note that the expansion stands for a classical field, using a quantized electromagnetic field would require additional features [12].

For a known distribution of scatterers, the operator $\hat{G}$ is the Green's function of the multiply scattering system for polarized waves. If the functions $\mathcal{G}, \mathcal{T}_{s s}(\omega, \theta)$ (defined in Secs. III and IV, respectively) and the operator $\hat{A}_{\text {spin }}$ are known, Eq. (27) for $\hat{G}$ solves the problem. But usually we are interested in disordered systems, for which the exact position of scatterers is unknown. It will thus be impossible to make a prediction depending on the particular realization of disorder, but it is possible to average over all such realizations (ensemble average) and to write a generalized GreenDyson equation for the average operators. We formulate the results using the simplest possible model of disorder. To perform the average over disorder, we neglect the volume of the scatterers and consider that each position $\mathbf{r}_{i}$ is uniformly distributed in the volume $\mathcal{V}$. The contribution of disorder in Eq. (27) comes exclusively from the averaging of the operator $\hat{T} \hat{\varrho}$. We introduce the self-energy $\hat{\Sigma}(\omega)$

$$
\hat{\Sigma}(\omega)=\overline{\hat{T}(\omega) \hat{\varrho}(\mathbf{r})} \text {. }
$$

The line denotes the average over all positions for all the scatterers, which we will explicitly define now for the calculation of the average density. The average of an operator $\overline{\hat{O}}$ is simply defined for arbitrary $\langle 1|$ and $|2\rangle$ by $\langle 1|\hat{\hat{O}}| 2\rangle=\overline{\langle 1|\hat{O}| 2\rangle}$. If the scatterers all have the same operator $\hat{T}(\omega)$, the selfenergy becomes

$$
\begin{aligned}
\hat{\Sigma}(\omega) & =\overline{\hat{T}(\omega) \hat{\varrho}(\mathbf{r})}=\hat{T}(\omega) \int_{\mathcal{V}} \frac{d \mathbf{r}_{1}}{\mathcal{V}} \cdots \int_{\mathcal{V}} \frac{d \mathbf{r}_{N}}{\mathcal{V}} \sum_{i=1}^{N} \delta^{(3)}\left(\mathbf{r}_{i}-\mathbf{r}\right) \\
& =\hat{T}(\omega) \sum_{i=1}^{N} \frac{1}{\mathcal{V}}=\hat{T}(\omega) \frac{N}{\mathcal{V}} \equiv \rho \hat{T}(\omega),
\end{aligned}
$$

where $\rho$ is the mean density. Our approach of disorder is nonetheless naive, we have neglected the possibility that a trajectory visits several times the same scatterer and thereby omitted some possible correlations in the averaging procedure. The topic of correlations created by several visits to the same scatterer is addressed abundantly in the literature. The most remarkable result is that for a sufficiently small density of scatterers; the expression of the Green-Dyson equation remains the same, but with a modified self-energy expressed as an expansion in powers of $\rho$ [7]. The first order of the expansion is exactly the expression we have found in formula (28), which is an encouraging fact in favor of an equivalent expansion in our framework. Such an equivalent expansion would extend the domain of validity of our results to higher densities. We will address this question in our future work.

Let us call $\bar{G}$ the average Green's operator resulting from the averaging over disorder of the operator $\hat{G}$ defined in Eq. (27). In the case where all scattering events are independent, the average of the products involving several times $\hat{\Sigma}$ are the products of the averages; this is sometimes called the Twersky approximation [6]. The Born expansion (27) becomes, in this approximation, the series

$$
\bar{G}=\hat{G}_{0}+\hat{G}_{0} \hat{\Sigma} \hat{G}_{0}+\hat{G}_{0} \hat{\Sigma} \hat{G}_{0} \hat{\Sigma} \hat{G}_{0}+\cdots .
$$

We have so far expressed the functions only in the direct space of position and frames. Physical problems however are easier to formulate in the reciprocal space because convolutions become products. We denote by $|\mathbf{q}\rangle$ the reciprocal space representation of $|\mathbf{r}\rangle$ and we have the well-known contraction

$$
\langle\mathbf{r} \mid \mathbf{q}\rangle=e^{\mathrm{iq} \cdot \mathbf{r}},
$$

which gives, together with the closure relation $\int d^{3} \mathbf{r}|\mathbf{r}\rangle\langle\mathbf{r}|$ $=\hat{1}$, the fundamental relations for the Fourier transform [47]. Similarly, we use the representation in reciprocal time that has the same properties as the reciprocal space.

As the system is invariant along time translations, the operators $\hat{T}, \hat{G}_{0}$, and $\bar{G}$, noted as $\hat{O}$, depend on one frequency $\omega$. Similarly, in the average medium obtained after integration over disorder, the translational invariance is restored; the operators only depend on one vector q. We introduce the notation $\hat{O}(\omega, \mathbf{q})$ for operators acting on the Hilbert space made of the elements $|P, s\rangle$, so that $\langle\mathbf{q}|\hat{O}(\omega)| \mathbf{q}\rangle$ $=\hat{O}(\omega, \mathbf{q}) \delta^{(3)}\left(\mathbf{q}^{\prime}-\mathbf{q}\right)$. The representation in reciprocal space for rotation and spin has different properties and is presented in the next section using an expression equivalent to Eq. (31).

The Born expansion written using the operators $\hat{\Sigma}(\omega)$, $\hat{G}_{0}(\mathbf{q}, \omega)$, and $\bar{G}(\mathbf{q}, \omega)$ reduces to

$$
\begin{aligned}
\bar{G}(\mathbf{q}, \omega)= & \hat{G}_{0}(\mathbf{q}, \omega)+\hat{G}_{0}(\mathbf{q}, \omega) \hat{\Sigma}(\omega) \hat{G}_{0}(\mathbf{q}, \omega) \\
& +\hat{G}_{0}(\mathbf{q}, \omega) \hat{\Sigma}(\omega) \hat{G}_{0}(\mathbf{q}, \omega) \hat{\Sigma}(\omega) \hat{G}_{0}(\mathbf{q}, \omega)+\cdots
\end{aligned}
$$

The operator $\hat{\Sigma}(\omega) \hat{G}_{0}(\omega, \mathbf{q})$ has a norm $\simeq \rho \sigma_{\text {ext }} q^{-1}$ and is therefore smaller than 1 for large $q$ or sufficiently small density $\rho$. Consequently, the series $\Sigma_{n}\left[\hat{\Sigma}(\omega) \hat{G}_{0}(\omega, \mathbf{q})\right]^{n}$ con- 
verges and we can rewrite the expansion (32) in a selfconsistent way

$$
\bar{G}(\mathbf{q}, \omega)=\hat{G}_{0}(\mathbf{q}, \omega)+\hat{G}_{0}(\mathbf{q}, \omega) \hat{\Sigma}(\omega) \bar{G}(\mathbf{q}, \omega) .
$$

Equation (33) is a generalized Green-Dyson equation for the directional Green's operators. The convergence of the series occurs when $q \ell>1$, where $\ell \equiv 1 / \rho \sigma_{\text {ext }}$ is the extinction mean free path. The theory described by the generalized Dyson equation does not apply to situations, where $q \ell \leq 1$, because scatterers are not in the far field of each other anymore. In the next section, we introduce the rotational harmonics transform on the variable $P$ to solve Eq. (33).

In the expression of the free Green's operator $\hat{G}_{0}(\omega, \mathbf{q})$ [Eq. (14)], the factor $\delta_{\perp}\left(D P^{-1}\right)$ modifies the usual expression of the Green's function $\sim 1 /\left(\frac{\omega^{2}}{c^{2}}-\mathbf{q}^{2} \pm i \eta\right)$, where the quantity $\eta$ is a infinitesimally small positive quantity. Using the identity

$$
\int d^{3} \mathbf{r} \frac{e^{\mathrm{i} \omega r / c}}{4 \pi r} e^{-\mathrm{i} \mathbf{q} \cdot \mathbf{r}} \delta_{\perp}\left(D P^{-1}\right)=\frac{1}{4 \pi} \frac{1}{\left(\frac{\omega}{c}-\mathbf{q} \cdot P \hat{\mathbf{z}}\right)^{2}},
$$

we get the Fourier transform

$$
\begin{aligned}
\left\langle P^{\prime}, s^{\prime}\left|\hat{G}_{0}^{R, A}(\omega, \mathbf{q})\right| P, s\right\rangle & \\
= & \frac{1}{\left(\frac{\omega}{c}-\mathbf{q} \cdot P \hat{\mathbf{z}}\right)^{2} \pm i \eta}\left\langle s^{\prime}\left|\hat{G}_{\text {spin }}(\omega)\right| s\right\rangle\left\langle P^{\prime} \mid P\right\rangle .
\end{aligned}
$$

The $R, A$ superscript stands for the retarded and advanced Green's operator. As we have already mentioned, the scalar theory must be retrieved if we sum our expressions over $P$ and $s$. Here we can check that Eq. (34) integrated over $P$ and summed up on $s$ yields formally

$$
\sum_{s=-S}^{S} \int_{\mathrm{SO}(3)} d P \frac{1}{\left(\frac{\omega}{c}-\mathbf{q} \cdot P \hat{\mathbf{z}}\right)^{2}} e^{\mathrm{i} s \psi} \Psi_{s}(\mathbf{q}, \omega)=\frac{1}{\frac{\omega^{2}}{c^{2}}-\mathbf{q}^{2}} \Psi_{0}(\mathbf{q}, \omega) .
$$

The regularization by the infinitesimal imaginary term $\pm i \eta$ can be performed on the right-hand side term of Eq. (35) so that we retrieve the usual Green's function for the field component with spin $s=0$. The superposition of all partial directional fields cancels out because of the phases of all the spins with $s \neq 0$. The final result depends on $q=|\mathbf{q}|$ : the directivity of the directional Green's operator (34) is lost. We call the expression $(\omega / c-\mathbf{q} \cdot P \hat{\mathbf{z}})^{-2}$ the directional factor of the Green's operator.

We can perform the same integration as in formula (35) on each operator $\hat{G}_{0}(\omega, \mathbf{q})$ in formula (33). We retrieve the scalar Green-Dyson equation $\bar{G}(\omega, q)=\mathcal{G}(\omega, q)$ $+\mathcal{G}(\omega, q) \Sigma(\omega) \bar{G}(\omega, q)$ with

$$
\Sigma(\omega)=\frac{1}{2} \rho \int_{0}^{\pi} \sin \theta d \theta \mathcal{T}_{00}(\omega, \theta) .
$$

The Green's operator $\bar{G}$ does not necessarily act separately on $|\mathbf{r}, t\rangle$ and $|P, s\rangle$. In optics, for instance, the Faraday effect in a medium with Verdet constant $V$ creates a phase shift $s V \mathbf{B} \cdot \mathbf{p}$. In this case, provided that the spin is conserved during propagation, Eq. (34) is modified and the Green's operator $\hat{G}_{0}$ becomes

$$
\begin{aligned}
& \left\langle P^{\prime}, s^{\prime}\left|\hat{G}_{0}^{R, A}(\mathbf{q}, \omega)\right| P, s\right\rangle \\
& \quad=\frac{1}{\left[\frac{\omega}{c}+(s V \mathbf{B}-\mathbf{q}) \cdot P \hat{\mathbf{z}}\right]^{2} \pm i \eta}\left\langle s^{\prime}\left|\hat{G}_{\mathrm{spin}}(\omega)\right| s\right\rangle\left\langle P^{\prime} \mid P\right\rangle .
\end{aligned}
$$

The separation of the paper into two parts to explain the harmonic transforms is intended only to keep the explanations simple. The Fourier transform presented in this section and the rotational harmonics transform that we introduce in the next section could have been presented simultaneously. This is actually mandatory in the case of a spin-orbit coupling involving $|P, s\rangle$ and $\mathbf{q}$ like the Faraday effect, as illustrated by formula (36).

\section{ROTATIONAL HARMONICS REPRESENTATION}

The linear representation of compact Lie groups provides a harmonical analysis on the group $\mathrm{SO}(3)$, which is the equivalent of Fourier series for periodic functions. These harmonical analysis is different from the Fourier transform because $\mathrm{SO}(3)$ is a compact noncommutative Lie group. For our purpose, there is no need to know this mathematical theory in detail. Some formulas used in our theory are presented in Appendix A.

The reciprocal representations are labeled by a main index $l \geq 0$, which can be an integer or a half-integer and two subindices $m$ and $n$, taking the values $-l,-l+1, \ldots, l$. We denote them by $\left|\begin{array}{c}l \\ m n\end{array}\right\rangle$ and we have

$$
\left\langle\left. R\right|_{m n} ^{l}\right\rangle=i^{n-m} \sqrt{2 l+1} e^{\mathrm{i} m \phi} d_{m, n}^{l}(\cos \theta) e^{\mathrm{i} n \psi},
$$

with $\phi, \theta$, and $\psi$ the Euler angles of $R$. Plots of the first $d_{m, n}^{l}$ function are displayed in Fig. 4. The closure relation,

$$
\sum_{l=\Lambda}^{\infty} \sum_{m=-l}^{l} \sum_{n=-l}^{l}\left|{ }_{m n}^{l}\right\rangle\left\langle{ }_{m n}^{l}\right|=\hat{1},
$$

is useful to obtain most of the result provided in the present work. We call the function $\left\langle\left. R\right|_{m n} ^{l}\right\rangle$ the rotational harmonics (the misleading terminology "generalized spherical harmonics" is often used). These functions are normalized Wigner $D$ functions $[18,43]$. The indices of the sums on $l, m$, and $n$ in Eq. (38) have steps equal to $1 . \Lambda$, the lowest order of $l$, depends on the spin $S$ of the wave and takes the value 0 or $1 / 2$ if the spin $S$ is an integer or a half-integer, respectively. It follows that the indices $l, m$, and $n$ are either all integers or all half-integers depending on $S$. For a given $l$ (the order of the harmonics), there are $(2 l+1)^{2}$ orthogonal functions, which seems a lot. We will see that for $l \geq S$, only $(2 S$ $+1)(2 l+1)$ harmonics components have to be taken into account. The functions $d_{m, n}^{l}$ are defined from $Z Y Z$ Euler angles 
and are real valued; their full expression is given in Eq. (A1). We have added the unconventional factor $i^{n-m}$ to obey the identity $\left\langle\left. R\right|_{m n} ^{l}\right\rangle^{*}=\left\langle\left. R^{-1}\right|_{n m} ^{l}\right\rangle$ [that is we have suppressed the factor $(-1)^{n-m}$ in this latter relation].

The rotational dependence of a state can be expressed as a function of the frame $P$ with the bracket notation $\langle P \mid \Psi\rangle$ or as a function of rotational harmonics indices $\left\langle_{m n}^{l} \mid \Psi\right\rangle$. Our computation uses this latter representation because it is related to the spin in a fundamental way. Let us use the closure relation of the rotation representation $\int_{\mathrm{SO}(3)} d R|R\rangle\langle R|=\hat{1}$ to get the expression

$$
\left\langle{ }_{m n}^{l}, s \mid \Psi\right\rangle=\int_{\mathrm{SO}(3)} d R\left\langle{ }_{m n}^{l}, s \mid R, s\right\rangle\langle R, s \mid \Psi\rangle .
$$

According to relations (4) and (37), the integration over the spin angle just leaves a factor $\delta_{n s}$, stating that the representation for a spin eigenvalue $s$ involves only the kets $\left|\begin{array}{c}l \\ m s\end{array}\right\rangle$. In the theory of multiple scattering, $S$ is fixed and there is only one species of particle involved. It means that in the Hilbert space of physical states, the equality $\left.\left.\right|_{m n} ^{l}\right\rangle \otimes|s\rangle=\left|{ }_{m s}^{l}\right\rangle \otimes|s\rangle \delta_{n s}$ is valid and the $n$ index can be left out without any restriction on the physical content of the theory.

We use the rotational harmonics to expressed the products of the operators used in the generalized Dyson Eq. (33),

$$
\begin{aligned}
& \left\langle\begin{array}{c}
l^{\prime} \\
m^{\prime} s^{\prime}
\end{array}\left|\bar{G}^{R, A}(\mathbf{q}, \omega)\right| \begin{array}{c}
l \\
m s
\end{array}\right\rangle=\left\langle\begin{array}{c}
l^{\prime} \\
m^{\prime} s^{\prime}
\end{array}\left|\hat{G}_{0}^{R, A}(\mathbf{q}, \omega)\right| \begin{array}{c}
l \\
m s
\end{array}\right\rangle \\
& +\sum_{\sigma=-S}^{S} \sum_{L=\Lambda}^{\infty} \sum_{M=-L}^{L} \sum_{\sigma^{\prime}=-S}^{S} \sum_{L^{\prime}=\Lambda}^{\infty} \sum_{M^{\prime}=-L^{\prime}}^{L^{\prime}} \\
& \left\langle\begin{array}{c}
l^{\prime} \\
m^{\prime} s^{\prime}
\end{array}\left|\hat{G}_{0}^{R, A}(\mathbf{q}, \omega)\right| \begin{array}{c}
L \\
M \sigma
\end{array}\right\rangle \\
& \times\left\langle\begin{array}{c}
L \\
M \sigma
\end{array}|\hat{\Sigma}(\omega)| \begin{array}{c}
L^{\prime} \\
M^{\prime} \sigma^{\prime}
\end{array}\right\rangle\left\langle\begin{array}{c}
L^{\prime} \\
M^{\prime} \sigma^{\prime}
\end{array}\left|\bar{G}^{R, A}(\mathbf{q}, \omega)\right| \begin{array}{c}
l \\
m s
\end{array}\right\rangle .
\end{aligned}
$$

Would we have used the representation in $\mathrm{SO}(3)$, we would have obtained integrals instead of the discrete sums. The sums over $L$ and $L^{\prime}$ are infinite. However, like in Fourier series, it is possible to truncate the sum because the brackets amplitudes decrease for large $L$ like $\alpha^{L}$ (for a certain $\alpha, 0$ $<\alpha<1$ ) such that high-order coefficients can be neglected. The sums on indices $L, M$, and $\sigma$ can be seen as sums over a single index. Equation (40) is therefore a discrete linear equation and is, up to a one-to-one transformation of $(L, M, \sigma)$ into a single index, a matrix equation. If the coefficients of $\hat{G}_{0}(\mathbf{q}, \omega)$ and $\hat{\Sigma}(\omega)$ are known, one can compute $\bar{G}(\mathbf{q}, \omega)$ with standard linear algebra and get a general solution for the transport of polarization in multiple scattering by inverting the matrix of $\hat{1}-\hat{G}_{0} \hat{\Sigma}$.

The directional Green's operator $\bar{G}$ describes the transport in an effective homogeneous medium. Paths statistics and Berry phases are included in this description, which represent an improvement over the usual effective Green's functions used in multiple-scattering theories. In the presence of several different kinds of scatterers, with densities $\rho_{i}$ and scattering matrices $\hat{T}_{i}$, we get an effective Green's operator by replacing $\hat{\Sigma}$ by $\Sigma_{i} \rho_{i} \bar{T}_{i}$. The scattering properties of the different scatterers are averaged and their respective weights are proportional to their respective densities. If the scatterers are not spherical and have random-independent orientations, the effective $\hat{T}$ matrix is the average over all orientations of the orientation-dependent $\hat{T}$ matrices. In particular, if the orientation probability is uniform, the average $\hat{T}$ matrix is equivalent to a spherical scatterer's $\hat{T}$ matrix and the spherical symmetry can be used as it is described in the next section.

To compute the rotational harmonics expansion of $\bar{G}$ up to order $L$, one needs to use approximately $(2 S+1) L^{2}$ triplets $(l, m, n)$ for the incoming frame, and as much for the outgoing frame. Therefore, the required number of rotational harmonics coefficients for the computation of the Green's operator scales like $(2 S+1)^{2} L^{4}$. The complexity of the inversion of the linear system (40) scales approximately like $L^{5}$.

\section{ROLE OF ROTATIONAL INVARIANCE}

We have considered in the previous sections the general case of a homogeneous medium invariant under translations, and we have decomposed the vectors of the threedimensional space into a radius part and an angular part to construct the general formalism for a directional Green's operator. A special and important case shows up when the medium and the scatterers are also both invariant under rotation. In the $\mathrm{SO}(3)$ representation, we have in this case for all frames $P$ and $P^{\prime}$ the equality already discussed in Sec. IV

$$
\left\langle P^{\prime}, s^{\prime}|\hat{\Sigma}(\omega)| P, s\right\rangle=\left\langle P^{-1} P^{\prime}, s^{\prime}|\hat{\Sigma}(\omega)| I, s\right\rangle .
$$

Using the closure relation (38), we get

$$
\begin{aligned}
& \left\langle P^{\prime}, s^{\prime}|\hat{\Sigma}(\omega)| P, s\right\rangle \\
& =\sum_{l=\Lambda}^{\infty} \sum_{m, n=-l}^{l}\left\langle P^{-1} P^{\prime},\left.s^{\prime}\right|_{m n} ^{l}\right\rangle\left\langle{ }_{m n}^{l} \hat{\Sigma}(\omega) \mid I, s\right\rangle .
\end{aligned}
$$

We expand the $\left\langle\left. P^{-1} P^{\prime}\right|_{m n} ^{l}\right\rangle$ thanks to formula (A12) and sum over $n$. After using formula (A13), we obtain

$$
\begin{aligned}
& \left\langle P^{\prime}, s^{\prime}|\hat{\Sigma}(\omega)| P, s\right\rangle \\
& =\sum_{l=\Lambda}^{\infty} \sum_{m, p=-l}^{l} \frac{1}{\sqrt{2 l+1}}\left\langle\left. P^{\prime}\right|_{p s^{\prime}} ^{l}\right\rangle\left\langle{ }_{m s^{\prime}}^{l} \hat{\Sigma}(\omega) \mid I, s\right\rangle\left\langle\left. P\right|_{p m} ^{l}\right\rangle .
\end{aligned}
$$

By identification of the terms of the right-hand sum with the rotational harmonics expansion in $P$ and $P^{\prime}$ of the left-hand term (A8), we get

$$
\begin{aligned}
\left\langle\underset{m^{\prime} s^{\prime}}{l^{\prime}}\left\langle|\hat{\Sigma}(\omega)|_{m s}^{l}\right\rangle\right. & =\frac{\delta_{l l^{\prime}} \delta_{m m^{\prime}}}{\sqrt{2 l+1}}\left\langle{ }_{s^{\prime} s}^{l}|\hat{\Sigma}(\omega)| I, s\right\rangle \\
& \equiv \frac{\delta_{l l^{\prime}} \delta_{m m^{\prime}}}{\sqrt{2 l+1}} \Sigma_{s^{\prime} s}^{l}(\omega) .
\end{aligned}
$$

If the medium and the scatterers are both invariant under rotations, the free Green's operator $\hat{G}_{0}$ and the Green-Dyson operator $\bar{G}$ are both independent from the reference frame. 
These operators depend on $\mathbf{q}$ and on the incoming and outgoing directions of propagation $P \hat{\mathbf{z}}$ and $P^{\prime} \hat{\mathbf{z}}$; a rotation of the reference frame acts simultaneously on these three vectors. Using the decomposition

$$
\mathbf{q}=q Q \hat{\mathbf{z}},
$$

where $Q$ is a rotation matrix and $q=|\mathbf{q}|$, we express the invariance of the Green's operators as

$$
\left\langle P^{\prime}, s^{\prime}\left|\hat{G}^{R, A}(\mathbf{q}, \omega)\right| P, s\right\rangle=\left\langle Q^{-1} P^{\prime}, s^{\prime}\left|\hat{G}^{R, A}(q, \omega)\right| Q^{-1} P, s\right\rangle,
$$

where $\hat{G}$ stands either for $\hat{G}_{0}$ or for $\bar{G}$. In Eq. (44), the operator $G$ depends only on $q$ and $\omega$. Using formula (34), one can even simplify further the Green's operators by defining

$$
\hat{G}_{0}^{R, A}(q, \omega)=\frac{c^{2}}{\omega^{2}} \hat{g}_{0}^{R, A}\left(\frac{c q}{\omega}\right),
$$

$$
\bar{G}^{R, A}(q, \omega)=\frac{c^{2}}{\omega^{2}} \bar{g}^{R, A}\left(\frac{c q}{\omega}\right) .
$$

As a consequence of formulas (14), we find a symmetry property for $\hat{g}_{0}$

$$
\left\langle\begin{array}{c}
l^{\prime} \\
m^{\prime} s^{\prime}
\end{array}\left|\hat{g}_{0}^{R, A}\right| \begin{array}{c}
l \\
m n
\end{array}\right\rangle \propto \delta_{m m^{\prime}} .
$$

The same symmetry has been observed for the $T$ matrix in Eq. (42) and we conclude that it will propagate to $\hat{g}$. A straightforward calculation shows that Eq. (33) takes the form

$$
\bar{g}^{R, A}\left(\frac{c q}{\omega}\right)=\hat{g}_{0}^{R, A}\left(\frac{c q}{\omega}\right)+\frac{c^{2}}{\omega^{2}} \hat{g}_{0}^{R, A}\left(\frac{c q}{\omega}\right) \hat{\Sigma}(\omega) \bar{g}^{R, A}\left(\frac{c q}{\omega}\right) .
$$

The nonvanishing rotational harmonics coefficients are related by

$$
\begin{aligned}
& \left\langle\begin{array}{c}
l_{m s^{\prime}} \\
l^{\prime}
\end{array} \bar{g}^{R, A}\left(\frac{c q}{\omega}\right) \mid \begin{array}{l}
l \\
m s
\end{array}\right\rangle \\
& \quad=\left\langle\underset{m s^{\prime}}{l^{\prime}}\left|\hat{g}_{0}^{R, A}\left(\frac{c q}{\omega}\right)\right|_{m s}^{l}\right\rangle+\frac{c^{2}}{\omega^{2}} \sum_{L=\Lambda}^{\infty} \frac{1}{\sqrt{2 L+1}} \sum_{\sigma=-S}^{S} \sum_{\sigma^{\prime}=-S}^{S}\left\langle\underset{m s^{\prime}}{l^{\prime}}\left|\hat{g}_{0}^{R, A}\left(\frac{c q}{\omega}\right)\right|_{m \sigma^{\prime}}^{L}\right\rangle \Sigma_{\sigma^{\prime} \sigma}^{L}\left\langle\underset{m \sigma}{L}\left|\bar{g}^{R, A}\left(\frac{c q}{\omega}\right)\right|_{m s}^{l}\right\rangle .
\end{aligned}
$$

Thanks to invariance under rotations, we have obtained a simplified formula for the generalized Green-Dyson equation. The matrices $\Sigma_{s^{\prime} s}^{l}$ are already known for several spherical scatterers, such as Mie scatterers. Formula (48) contains only three sums, two of which, over the indices $\sigma$ and $\sigma^{\prime}$, are finite sums and correspond to a matrix product. The expansion of $\bar{g}$ into rotational harmonics coefficients is therefore a single sum over a generalized index instead of a double sum as in Eq. (40). The computation of $\bar{g}$ requires to know the rotational harmonics coefficients of the $\Sigma$ matrix and of $\hat{g}_{0}$. The coefficients of $\hat{g}_{0}$ can be computed starting from expression (34),

$$
\begin{aligned}
& \left\langle\begin{array}{c}
l^{\prime} \\
m s^{\prime}
\end{array}\left|\hat{g}_{0}^{R, A}(x)\right| \begin{array}{c}
l \\
m s
\end{array}\right\rangle \\
& \quad=i^{s^{\prime}-s} \sqrt{l+\frac{1}{2}} \sqrt{l^{\prime}+\frac{1}{2}} \int_{-1}^{1} d \mu \frac{d_{m s}^{l}(\mu) d_{m s^{\prime}}^{l^{\prime}}(\mu)}{(1-\mu x)^{2} \pm \mathrm{i} \eta} .
\end{aligned}
$$

For arguments $x>1$, there is a pole at $1 / x$ in the integral (49), which has the same physical origin as the pole of the Green's function in a scalar representation.

\section{CONCLUSION}

We have presented a model for the transport of a polarized wave in a complex medium containing anisotropies. In our approach, the wave is represented in the particle picture of quantum mechanics; the transport of the wave is described as a superposition of trajectories with attached probabilities. The polarization of the wave follows from the spin of the particles. We express the amplitudes of the spin eigenstates in local frames. A local frame is a rotation matrix, where the third axis is colinear with the momentum of the particle. Local frames form a convenient setting for polarized waves because the phases of the spin eigenstates of a particle are related to each other by frame-dependent factors. Based on the local frame representation, we have established a theory for the multiple scattering of particles with spin and we have obtained a generalized Green-Dyson equation, which takes into account the three-dimensional nature of the transport. One can realize the three-dimensional nature of the formalism by noticing that the Berry phase, by essence a threedimensional concept, naturally emerges from our equations.

We have suggested to solve the generalized Green-Dyson equation using rotational harmonics, which transforms the convolutions on $\mathrm{SO}(3)$ into matrix products. The solutions of the generalized Green-Dyson equation are obtained by linear algebra operations. The local frame description is able to take into account several circular anisotropies, such as birefringence and dischroism, Faraday effect, and anisotropic scattering. In the special and important case where the scatterers and the medium are both invariant under rotations, the generalized Green-Dyson equation takes a simpler form and should be solvable with reasonably light numerical power. 
Linear birefringence and dichroism can also be described in the theory but require a more complex mathematical treatment, which was not presented here for the sake of simplicity. Beyond the theory of multiple scattering, we believe that the generalized Green-Dyson operator is a useful object for the statistical study of random three-dimensional paths, thanks to its ability to perform path integrals of directiondependent functionals.

The computation of the solution of the generalized GreenDyson equation using rotational harmonics is probably the most efficient because it reproduces the properties of the Fourier transform used to solve the usual Green-Dyson equation. On one hand, as the rotational harmonics form a discrete basis of the algebra on $\mathrm{SO}(3)$, one only needs to compute for each value of $c q / \omega$ a discrete set of coefficients. On the other hand, the maximum order $L$ one has to use to get a physically relevant result grows with the strongest anisotropy of the system. Despite the simplicity of the expression of the solutions, it is not conceivable to draw the calculations analytically in general. The numerical difficulties lie in two points: the inversion of the linear system (40) or (48) and the expression of the operator $\hat{g}_{0}(49)$.

We have neglected the interactions between scatterers, which can create correlations between their positions. Interactions can be handled by the introduction of the paircorrelation function (or a structure factor), which would enter as a factor in the second-order term of the Born expansion [44]. In terms of higher order $n$ of the Born expansion, one should manipulate $n$-points correlation functions, which introduce a supplementary difficulty. Much less is known concerning the interacting scatterers than concerning the selfenergy. We also considered that the medium is globally invariant under translation and that the density of scatterers is homogeneous, which excludes small size systems and leads out the effects of boundaries. Our theory concerns the bulk of a system, but experiments are usually carried out from the edges. It is therefore an important issue to investigate the role of the boundaries.

As such, our work should rather be considered as a step toward a full theory of polarization transport in the presence of anisotropies. It may nonetheless already lead to experimental applications and investigations in complex media. On a more general point of view, our work suggests that polarized waves in complex media contain more information concerning anisotropies than scalar waves and that polarization is therefore a relevant supplementary observable for the investigation of anisotropic systems. Our future work will be dedicated to improve the domain of application of the presented framework to denser interacting or quantum systems. First of all, the expression of the self-energy expansion in powers of the density should be extended up to higher orders and the formalism for intensity diagrams should be developed.

\section{ACKNOWLEDGMENTS}

The author is indebted to T. Champel, S. Skipetrov, and B. van Tiggelen for their sharp comments and productive suggestions concerning this work. This work was partially sup- ported by the ANR Grant No. 08-JCJC-0066 SISDIF.

\section{APPENDIX A: THE ROTATIONAL HARMONICS}

The rotational harmonics analysis is similar to the Fourier analysis for periodic functions. It is the direct application of the theorem of Peter and Weyl [45] to the representations of the group $\mathrm{SO}(3)$. Peter-Weyl theorem states that any complex function $f$ defined on $\mathrm{SO}(3)$ may be developed into rotational harmonics series. The representations of $\mathrm{SO}(3)$ were introduced in physics by Wigner [18] but remain confidential in physical theories mostly because in spinless theories the rotational harmonics simplify to spherical harmonics. An important application in physics was to establish WignerEckart's theorem $[18,46]$. [One can notice that the Eq. (42) is a particular case Wigner-Eckart's theorem.] It was shown in Sec. VII that the set of rotational harmonics useful in the problem of scattering of spin particles depends on the spin $S$. More precisely, it depends whether $S$ is an integer or a halfodd integer. The rotational harmonics with a half-odd integer main index stand for fermions, while the ones with an integer main index stand for bosons.

The rotational harmonics are given by formula (37) and

$$
\begin{aligned}
d_{m n}^{l}(\cos \theta)= & \sqrt{(l-m) !(l+m) !(l-n) !(l+n) !} \\
& \times \sum_{p=\max \{0, m-n\}}^{\min \{l+m, l-n\}}(-1)^{p} \\
& \times \frac{\left(\cos \frac{\theta}{2}\right)^{2 l+m-n-2 p}\left(\sin \frac{\theta}{2}\right)^{n-m+2 p}}{(l+m-p) ! p !(n-m+p) !(l-n-p) !} .
\end{aligned}
$$

Example of plots are displayed in Fig. 4. Remark that the arguments of factorials and the powers are integers, in both cases where $l, m$, and $n$ are integers or half-integers. The computations of the set of $d_{m n}^{l}$ functions are simplified by the symmetries

$$
\begin{aligned}
d_{m, n}^{l}(\cos \theta) & =(-1)^{l+m} d_{m,-n}^{l}(-\cos \theta), \\
& =(-1)^{l+m} d_{m,-n}^{l}[\cos (\pi-\theta)], \\
& =(-1)^{m-n} d_{-m,-n}^{l}(\cos \theta), \\
& =(-1)^{m-n} d_{n, m}^{l}(\cos \theta) .
\end{aligned}
$$

One can also define the rotational harmonics from the spherical harmonics by means of an operator introduced in references [19]. This result is sketched in Appendix B.

In our notations, the harmonics expansion is written

$$
f(R)=\langle R \mid f\rangle=\sum_{l \geq \Lambda} \sum_{m, n=-l}^{l}\left\langle\left. R\right|_{m n} ^{l}\right\rangle\left\langle_{m n}^{l} \mid f\right\rangle,
$$

where the harmonics coefficients are 


$$
\left\langle{ }_{m n}^{l} \mid f\right\rangle=\int_{\mathrm{SO}(3)} d R\left\langle{ }_{m n}^{l} \mid R\right\rangle\langle R \mid f\rangle=\int_{\mathrm{SO}(3)} d R\langle R|{ }_{m n}^{l}{ }^{*} f(R) .
$$

Similarly, the operators can be expressed in the basis of the rotational harmonics,

$$
\begin{aligned}
\mathrm{O}\left(R^{\prime}, R\right)= & \left\langle R^{\prime}|\hat{O}| R\right\rangle=\sum_{l, l^{\prime} \geq \Lambda} \sum_{m, n=-l}^{l} \sum_{m^{\prime} n^{\prime}=-l^{\prime}}^{l^{\prime}}\left\langle{ }_{m^{\prime} n^{\prime}}|\hat{O}|_{m n}^{l}\right\rangle \\
& \times\left\langle R^{\prime} \mid{ }_{m^{\prime} n^{\prime}} l^{l^{\prime}}\right\rangle\left\langle\left. R\right|_{m n}\right\rangle^{l},
\end{aligned}
$$

where

$$
\left\langle\begin{array}{c}
l^{\prime} \\
m^{\prime} n^{\prime}
\end{array}|\hat{O}|_{m n}^{l}\right\rangle=\int_{\mathrm{SO}(3)} d R^{\prime} \int_{\mathrm{SO}(3)} d R O\left(R^{\prime}, R\right)\left\langle\left. R\right|_{m^{\prime} n^{\prime}} ^{l^{\prime}}\right\rangle^{*}\left\langle\left. R\right|_{m n} ^{l}\right\rangle \text {. }
$$

The product of operators $\hat{C}=\hat{A} \hat{B}$ is expressed in direct space as a convolution

$$
\left\langle R^{\prime}|\hat{C}| R\right\rangle=\int_{\mathrm{SO}(3)}\left\langle R^{\prime}|\hat{A}| X\right\rangle\langle X|\hat{B}| R\rangle d X,
$$

and in harmonics space as a series

$$
\left\langle\begin{array}{c}
l^{\prime} \\
m^{\prime} n^{\prime}
\end{array}|\hat{C}|_{m n}^{l}\right\rangle=\sum_{L=\Lambda}^{\infty} \sum_{M, N=-L}^{L}\left\langle\begin{array}{c}
l^{\prime} \\
m^{\prime} n^{\prime}
\end{array}|\hat{A}|_{M N}^{L}\right\rangle\left\langle{ }_{M N}^{L}|\hat{B}|_{m n}^{l}\right\rangle .
$$

The sum over $M$ and $N$ is the usual matrix product. In our calculations, we also use the relations

$$
\begin{aligned}
\sum_{p}\left\langle\left. R_{1}\right|_{m p} ^{l}\right\rangle\left\langle\left. R_{2}\right|_{p n} ^{l}\right\rangle & =\sqrt{2 l+1}\left\langle\left. R_{1} R_{2}\right|_{m n} ^{l}\right\rangle, \\
\left\langle\left. R^{-1}\right|_{m n} ^{l}\right\rangle & =\left\langle\left. R\right|_{n m} ^{l}\right\rangle^{*} .
\end{aligned}
$$

It is also interesting to note that we have the ParsevalPlancherel formulas,

$$
\begin{aligned}
\langle f \mid f\rangle & =\int_{\mathrm{SO}(3)}|f(R)|^{2} d R=\sum_{l=\Lambda}^{\infty} \sum_{m, n=-l}^{l}\left|\left\langle{ }_{m n}^{l} \mid f\right\rangle\right|^{2}, \\
\int d R \int d R^{\prime}\left|\left\langle R^{\prime}|\hat{O}| R\right\rangle\right|^{2} & \\
& =\sum_{l, l^{\prime}=\Lambda}^{\infty} \sum_{m, n=-l}^{l} \sum_{m^{\prime}, n^{\prime}=-l^{\prime}}^{l^{\prime}}\left|\left\langle{ }_{m^{\prime} n^{\prime}}|\hat{O}|_{m n}^{l}\right\rangle\right|^{2} .
\end{aligned}
$$

\section{APPENDIX B: ROTATIONAL HARMONICS UNDER OTHER NAMES}

In this appendix, we compile the relations between the rotational harmonics and other special functions used in literature under several names and with a large variety of normalizations. This appendix is intended to allow those famil- iar with one the numerous forms of the rotational harmonics to comprehend our presentations from a wider point of view. Conventionally, the argument $\cos \theta$ may be written $\mu$.

Wigner introduced the rotational harmonics arranged in matrices called Wigner's $D$ matrices of order $l$ [18]. The elements of $D(l)$ are given by

$$
D_{m^{\prime} m}^{l}(\phi, \theta, \psi)=e^{-\mathrm{i} m^{\prime} \phi} d_{m^{\prime} m}^{l}(\cos \theta) e^{-\mathrm{i} m \psi} .
$$

\section{Functions $\pi$ and $\tau$ used in the Mie expansion}

The well-known Mie theory for spherical dielectric scatterers provides the general solution of the scattering of a plane wave by a dielectric sphere of arbitrary size and refraction index $[1,2]$. Forward scattering appears for large spheres of radius $a \gg 2 \pi / \lambda$; this is sometimes called the Mie effect. The scatterers are spherical, so they are invariant under rotations and the $T$ matrix is thus of the form of Eq. (42). Written in the spin eigenstates basis (also called circular polarization basis), the $T$-matrix coefficients take the form

$$
\mathcal{T}_{s s^{\prime}}(\omega, \theta)=\frac{2 i \pi c}{\omega} i^{s}\left[S_{1}(\theta)+s s^{\prime} S_{2}(\theta)\right] \quad s, s^{\prime}= \pm 1,
$$

with the coefficients $S_{1}(\theta)$ and $S_{2}(\theta)$ as defined by van de Hulst [4].

Mie theory provides the expansion of the coefficients $S_{1}$ and $S_{2}$ into series of terms involving special functions referred as $\pi_{n}$ and $\tau_{n}$ in [4] and in many other textbooks. The coefficients of the expansion are called $a_{n}$ for $S_{1}$ and $b_{n}$ for $S_{2}$. In the circular polarization basis, we obtain the main coefficients as combination of $\pi_{n}$ and $\tau_{n}$,

$$
\begin{aligned}
& S_{1}(\theta)+S_{2}(\theta)=\sum_{n=1}^{\infty}(2 n+1) \frac{a_{n}+b_{n}}{2} \frac{\pi_{n}(\cos \theta)+\tau_{n}(\cos \theta)}{n(n+1)}, \\
& S_{1}(\theta)-S_{2}(\theta)=\sum_{n=1}^{\infty}(2 n+1) \frac{a_{n}-b_{n}}{2} \frac{\pi_{n}(\cos \theta)-\tau_{n}(\cos \theta)}{n(n+1)} .
\end{aligned}
$$

The functions $\pi_{l}(\mu)$ and $\tau_{l}(\mu)$ are defined by $\pi_{0}=0, \pi_{1}=1$, and

$$
\begin{gathered}
\pi_{l+1}(\mu)=\frac{2 l+1}{l} \mu \pi_{l}(\mu)-\frac{l+1}{l} \pi_{l-1}(\mu), \\
\tau_{l}(x)=l \mu \pi_{l}(\mu)-(l+1) \pi_{l-1}(\mu) .
\end{gathered}
$$

As it was shown by Domke [20], we have the relations

$$
\begin{aligned}
& \pi_{l}(\mu)=l(l+1)\left[d_{1,1}^{l}(\mu)+d_{1,-1}^{l}(\mu)\right], \\
& \tau_{l}(\mu)=l(l+1)\left[d_{1,1}^{l}(\mu)-d_{1,-1}^{l}(\mu)\right] .
\end{aligned}
$$

[In [5], the functions $\pi$ and $\tau$ are defined with the same equations, without the factor $l(l+1)$.]

It appears that the Mie expansion is nothing but the rotational harmonics expansion for spins $s= \pm 1$ of a spherical 
scatterer's $\hat{T}$ matrix. In the low-density approximation used in the paper, the self-energy matrix defined in Eq. (42) is then

$$
\Sigma_{1, \pm 1}^{l}=-\Sigma_{-1, \mp 1}^{l}=2 i \pi(2 l+1) \frac{\rho c}{\omega} \frac{a_{l} \pm b_{l}}{2} .
$$

One should not be surprised because Mie's result comes from the resolution of Maxwell's equations, which are vectorial, thus by nature concerning fields of spin $S=1$.

\section{Vector spherical harmonics}

In electromagnetism, the introduction of spherical harmonics is motivated by the possibility of multipole expansion to solve several problems [3,5]. The multipolar expansion of a vector field can be expressed in terms of "vector spherical harmonics." There does not seem to be a standard notation for these vectors. In Ref. [3], one finds the definition of $\mathbf{X}_{l m}(\theta, \phi)$, while in Ref. [5] we have

$$
\begin{gathered}
\mathbf{Y}_{l m}^{(e)}(\mathbf{r})=\frac{1}{\sqrt{l(l+1)}} r \nabla Y_{l m}, \\
\mathbf{Y}_{l m}^{(m)}(\mathbf{r})=r \mathbf{X}_{l m}=\hat{\mathbf{r}} \times \mathbf{Y}_{l m}^{(e)}, \\
\mathbf{Y}_{l m}^{(o)}(\mathbf{r})=Y_{l m} \mathbf{r} .
\end{gathered}
$$

The spherical harmonics $Y_{l m}$ being given by

$$
Y_{l m}(\theta, \phi)=\sqrt{\frac{2 l+1}{4 \pi}} \sqrt{\frac{(l-m) !}{(l+m) !}} d_{m, 0}^{l}(\cos \theta) e^{\mathrm{i} m \phi},
$$

we have in spherical coordinates $(\hat{\mathbf{r}}, \hat{\theta}, \hat{\phi})$

$$
\begin{aligned}
\mathbf{Y}_{l m}^{(e)}(\mathbf{r})= & \frac{(-1)^{m} i^{l}}{\sqrt{l(l+1)}} \sqrt{\frac{2 l+1}{4 \pi}} \sqrt{\frac{(l-m) !}{(l+m) !}} \\
& \times \frac{e^{\mathrm{i} m \phi}}{\sin \theta}\left(\begin{array}{c}
\frac{1-\mu^{2}}{2}\left[d_{m, 1}^{l}(\mu)+d_{m,-1}^{l}(\mu)\right] \\
i m d_{m, 0}^{l}(\mu)
\end{array}\right) .
\end{aligned}
$$

Only the vector spherical harmonics with $m= \pm 1$ are used to solve electromagnetic problems. We find again that it is more natural to look for the solutions using the basis adapted to spin $S=1$ in electromagnetism.

\section{Generalized spherical harmonics}

In the work of Kuščer and Ribarič, "generalized spherical harmonics" have been introduced for the Stokes parameters describing polarized light [17]. Stoked parameters are intensities and therefore correspond to the amplitude of the wave field squared. This is why the rotational harmonics appear in this work and followers with a power of two [20,21,23]. In other words, the expansion involves the functions $d_{m, 0}^{l}$ and $d_{m, \pm 2}^{l}$. The spherical functions are, in these papers, noted $P_{m n}^{l}(\cos \theta)$ and are simply

$$
P_{m n}^{l}(\mu)=i^{n-m} d_{m n}^{l}(\mu)
$$

Our rotational harmonics $\left\langle R \mid{ }_{m n}^{l}\right\rangle$ only differ from these spherical functions by a normalization factor $\sqrt{2 l+1}$, which we introduced to simplify the expression of the convolution formulas.

\section{Spin-weighted spherical harmonics ${ }_{s} Y_{l}^{m}$}

Newman and Penrose introduced functions called the "spin-weighted spherical harmonics" to study gravitational radiations (which have a spin $S=2$ ) [19]. The spin-weighted functions are obtained after derivation of the spherical harmonics by the operator $\partial$ (read "thop") defined, for a spinweight $s$ function $f_{s}$, by

$$
\partial f_{s}(\theta, \phi)=-(\sin \theta)^{s}\left[\frac{\partial}{\partial \theta}+i \frac{1}{\sin \theta} \frac{\partial}{\partial \phi}\right](\sin \theta)^{-s} f_{s}(\theta, \phi) .
$$

The usual spherical harmonics are of spin-weight 0 and the spin-weighted spherical harmonics of spin $s$ are defined by $Y_{l m}(\theta, \phi)=\frac{1}{2} \sqrt{\frac{(l-s) !}{(l+s) !}} \partial^{s} Y_{l m}$ for $s>0$ and ${ }_{s} Y_{l m}(\theta, \phi)$ $=\frac{1}{2} \sqrt{\frac{(l-s) !}{(l+s) !}}(-\partial)^{s} Y_{l m}$ for $s<0$. We have the relation

$$
{ }_{s} Y_{l m}(\theta, \phi)=(-1)^{m+s} \sqrt{\frac{2 l+1}{4 \pi}} d_{m,-s}^{l}(\cos \theta) e^{\mathrm{i} m \phi}
$$

[1] M. Born and E. Wolf, Principles of Optics, 7th ed. (Cambridge University Press, New York, 1999).

[2] G. Mie, Ann. Phys. 330, 377 (1908).

[3] J. D. Jackson, Classical Electrodynamics, 2nd ed. (John Wiley and Sons, New York, 1972).

[4] H. C. Van de Hulst, Light Scattering by Small Particles (John Wiley and sons, New York, 1957).

[5] R. G. Newton, Scattering Theory of Waves and Particles
(Springer, New York, 1982).

[6] M. I. Mischchenko, L. D. Travis, and A. A. Lacis, Multiple Scattering of Light by Particles (Cambridge University Press, New York, 2006).

[7] M. C. W. van Rossum and T. M. Nieuwenhuizen, Rev. Mod. Phys. 71, 313 (1999).

[8] E. Akkermans and G. Montambaux, Mesoscopic Physics of Electrons and Photons (Cambridge University Press, New 
York, 2006).

[9] F. A. Pinheiro and B. A. van Tiggelen, J. Opt. Soc. Am. A Opt. Image Sci. Vis. 20, 99 (2003).

[10] G. Labeyrie, F. de Tomasi, J.-C. Bernard, C. A. Müller, C. Miniatura, and R. Kaiser, Phys. Rev. Lett. 83, 5266 (1999).

[11] T. Jonckheere, C. A. Müller, R. Kaiser, C. Miniatura, and D. Delande, Phys. Rev. Lett. 85, 4269 (2000).

[12] D. V. Kupriyanov, I. M. Sokolov, C. I. Sukenik, and M. D. Havey, Laser Phys. Lett. 3, 223 (2006).

[13] I. M. Sokolov, M. D. Kupriyanova, D. V. Kupriyanov, and M. D. Havey, Phys. Rev. A 79, 053405 (2009).

[14] G. Labeyrie, D. Delande, C. A. Müller, C. Miniatura, and R. Kaiser, Opt. Commun. 243, 157 (2004).

[15] L. Margerin, M. Campillo, and B. van Tiggelen, J. Geophys. Res. 105, 7873 (2000).

[16] H. Sato, Geophys. J. Int. 121, 523 (1995).

[17] I. Kuščer and M. Ribarič, J. Mod. Opt. 6, 42 (1959).

[18] E. P. Wigner, Group Theory and its Application to the Quantum Mechanics of Atomic Spectra (Academic Press, New York, 1959).

[19] E. T. Newman and R. Penrose, J. Math. Phys. 7, 863 (1966).

[20] H. Domke, Z. Meteo. 25, 357 (1975).

[21] C. E. Siewert, Astron. Astrophys. 109, 195 (1982).

[22] D. Lacoste, B. A. van Tiggelen, G. L. J. A. Rikken, and A. Sparenberg, J. Opt. Soc. Am. A Opt. Image Sci. Vis. 15, 1636 (1998).

[23] J. W. Hovenier and C. V. M. van der Mee, Astron. Astrophys. 128, 1 (1983).

[24] R. D. M. Garcia and C. E. Siewert, J. Quant. Spectrosc. Radiat. Transf. 36, 401 (1986).

[25] S. R. Pal and A. I. Carswell, Appl. Opt. 24, 3464 (1985).

[26] A. H. Hielscher, A. A. Eick, J. R. Mourant, D. Shen, J. P.
Freyer, and I. J. Bigio, Opt. Express 1, 441 (1997).

[27] B. D. Cameron, M. J. Raković, M. Mehrübeoğlu, G. W. Kattawar, S. Rastegar, L. V. Wang, and G. L. Coté, Opt. Lett. 23 485 (1998).

[28] V. Rossetto and A. C. Maggs, Eur. Phys. J. B 29, 323 (2002).

[29] M. V. Berry, Proc. R. Soc. London, Ser. A 392, 45 (1984).

[30] Geometric Phases in Physics, edited by A. Shapere and F. Wilczek (World Scientific, Singapore, 1989).

[31] A. Tomita and R. Y. Chiao, Phys. Rev. Lett. 57, 937 (1986).

[32] M. V. Berry, J. Mod. Opt. 34, 1401 (1987).

[33] R. Bhandari, Phys. Rep. 281, 1 (1997).

[34] A. C. Maggs and V. Rossetto, Phys. Rev. Lett. 87, 253901 (2001).

[35] M. Jacob and G. C. Wick, Ann. Phys. 7, 404 (1959).

[36] P. G. Kwiat and R. Y. Chiao, Phys. Rev. Lett. 66, 588 (1991).

[37] J. Samuel and R. Bhandari, Phys. Rev. Lett. 60, 2339 (1988).

[38] F. B. Fuller, Proc. Natl. Acad. Sci. U.S.A. 68, 815 (1971).

[39] V. Rossetto and A. C. Maggs, Phys. Rev. Lett. 88, 089801 (2002).

[40] L. D. Landau and E. M. Lifshitz, Mechanics, 3rd ed. (Butterworth-Heinemann, Oxford, 1993).

[41] M. M. G. Krishna, J. Samuel, and S. Sinha, J. Phys. A 33, 5965 (2000).

[42] V. Rossetto, Europhys. Lett. 69, 142 (2005).

[43] I. M. Gel'fand and Z. Y. Šapiro, Am. Math. Soc. Transl. 2, 207 (1956).

[44] S. Fraden and G. Maret, Phys. Rev. Lett. 65, 512 (1990).

[45] F. Peter and H. Weyl, Math. Ann. 97, 737 (1927).

[46] C. Eckart, Rev. Mod. Phys. 2, 305 (1930).

[47] There are several presentations of the Fourier transform, with different normalizations. Here, the normalization consistent with our formulas is $\int d^{3} \mathbf{q}|\mathbf{q}\rangle\langle\mathbf{q}|=(2 \pi)^{3} \hat{1}$. 